\title{
Concentration and velocity statistics of inertial particles in upward and downward pipe flow
}

\author{
Goes Oliveira, J.L.; van der Geld, C.W.M.; Kuerten, J.G.M.
}

Published in:

Journal of Fluid Mechanics

DOI:

10.1017/jfm.2017.289

Published: 10/07/2017

Document Version

Accepted manuscript including changes made at the peer-review stage

Please check the document version of this publication:

- A submitted manuscript is the author's version of the article upon submission and before peer-review. There can be important differences between the submitted version and the official published version of record. People interested in the research are advised to contact the author for the final version of the publication, or visit the DOI to the publisher's website.

- The final author version and the galley proof are versions of the publication after peer review.

- The final published version features the final layout of the paper including the volume, issue and page numbers.

Link to publication

Citation for published version (APA):

Goes Oliveira, J. L., van der Geld, C. W. M., \& Kuerten, J. G. M. (2017). Concentration and velocity statistics of inertial particles in upward and downward pipe flow. Journal of Fluid Mechanics, 822, 640-663. DOI:

10.1017/jfm.2017.289

\section{General rights}

Copyright and moral rights for the publications made accessible in the public portal are retained by the authors and/or other copyright owners and it is a condition of accessing publications that users recognise and abide by the legal requirements associated with these rights.

- Users may download and print one copy of any publication from the public portal for the purpose of private study or research.

- You may not further distribute the material or use it for any profit-making activity or commercial gain

- You may freely distribute the URL identifying the publication in the public portal ?

Take down policy

If you believe that this document breaches copyright please contact us providing details, and we will remove access to the work immediately and investigate your claim. 


\title{
Concentration and velocity statistics of inertial particles in upward and downward pipe flow
}

\author{
J. L. G. Oliveira, ${ }^{\mathrm{a}, 1}$ C. W. M. van der Geld, ${ }^{\text {b,* }}$ and J. G. M. Kuerten ${ }^{\mathrm{a}, \mathrm{c}}$ \\ ${ }^{a}$ Department of Mechanical Engineering, Eindhoven University of Technology, P.O. Box 513, 5600 MB \\ Eindhoven, The Netherlands \\ ${ }^{b}$ Department of Chemical Engineering and Chemistry, Eindhoven University of Technology, The \\ Netherlands \\ ${ }^{\mathrm{c}}$ Faculty EEMCS, University of Twente, The Netherlands
}

Three-dimensional particle tracking velocimetry (3D-PTV) is applied to particle-laden turbulent pipe flows at Reynolds number 10300, based on the bulk velocity and the pipe diameter, for developed fluid flow and not fully developed flow of inertial particles that favors assessment of the radial migration of the inertial particles. Inertial particles with Stokes number ranging from 0.35 to 1.11 , based on the particle relaxation time and radial dependent Kolmogorov timescale, and ratio of the RMS fluid velocity to the terminal velocity of order 1 have been used. Core peaking of the concentration of inertial particles in up-flow and wall peaking in down-flow have been found. The difference of mean particle and Eulerian mean liquid velocity is found to decrease to about zero near the wall in both flow directions. Although the carrier fluid has all the characteristics of the corresponding turbulent single-phase flow, the Reynolds stress of inertial particles is different near the wall in up-flow. These findings are explained from preferential location of the inertial particles, with the aid of direct numerical simulations with the point particle approach.

1 Present address: Mobility Department, Federal University of Santa Catarina, Joinville/SC, 89218-000, Brazil. E-mail address: jorge.goes@ufsc.br

* Corresponding author (C.W.M. van der Geld): Tel.: +31 402472923; fax: +31 402475399. E-mail address: C.W.M.v.d.Geld@tue.nl 


\section{Introduction}

Turbulent dispersed two-phase flows are abundant in both industry and nature. Dispersion of pollutants in an urban environment, sediment transport and the fluidized catalytic cracking of carbohydrates are for example of major importance. The ability to predict migration of a dispersed phase with a different velocity than the carrier phase - either with numerical or analytical models - is required in a wide range of disciplines. A thorough understanding of the behavior of this type of dispersed two-phase flows in practice is essential for model development and facilitates scale-up of process equipment and improvement of mixing efficiencies. It is clear that a consistent set of experimental data under well-known conditions is needed to validate models. The present study aims at increasing our understanding and providing such a set of data. Before describing the main features of the approach followed, first previous experimental work and relevant theoretical studies are reviewed.

All turbulent flows in practice are inhomogeneous. Although experiments of any kind are useful to reveal the underlying physics of dispersed two-phase flows, experiments of inhomogeneous flows are therefore particularly significant. The most familiar example of such flows is turbulent flow in pipes. Although particle-laden flow in pipes has numerous engineering applications, only restricted classes of turbulent dispersed flows in pipes have been investigated, and to a limited extent. These will be reviewed first.

A significant number of experimental studies have been performed with small solid particles in wallbounded flows, pipes or channels; small here means with a size in the order of the Kolmogorov lengthscale or less. Most of the particles possessed a significant relaxation time-scale owing to high mass density ratios, as for solid particles in gas flows. The characteristics of both the carrier fluid phase and small solid particles were measured in turbulent wall-bounded flows by Tsuji \& Morikawa (1982), Tsuji, Morikawa \& Shiomi (1984), Kulick et al. (1994), Paris \& Eaton (2001), Kussin \& Sommerfeld (2002), Caraman et al. (2003), Yang \& Shy (2005), Benson, Tanaka \& Eaton (2005), and Borée \& Caraman (2005). In these studies, attempts were made to explain particle migration, preferential concentrations and turbulence modulation. So-called instantaneous realizations of the fluid velocity field, instantaneous 3D snapshots of the flow field, were combined in the analysis with considerations of particle inertia. Some of these analyses also considered effects of wake interaction, wall roughness and inter-particle and particle-wall collisions. The latter are of course only significant with high particle loadings. To avoid the need to investigate many different phenomena, it is wise to pay attention to flows with a low concentration of particles. Flows in which the collision frequency, breakage efficiency, agglomeration, reaction rates, deposition or entrainment of particles is essential will therefore not be addressed in the present study.

As opposed to the above small and heavy particles, near-neutrally buoyant particles with significant dimension have less often been investigated. In this case the particle diameter to Kolmogorov length-scale ratio is high, which induces a high relaxation time once again. An example is polystyrene particles in water flows. The dynamics of such particles in turbulent transport has been the subject of experimental research in more recent studies of Brown et al. (2009) and Volk et al. (2011). These studies concerned counter rotating disk flow with neutrally buoyant particles. The particle inertia is quite high owing to added mass and the particle size. At the expense of having large size particles, the relative velocity is kept limited by selecting a particle mass-density close to that of water. Calzavarini et al. (2009) developed a numerical model to predict features of neutrally buoyant particles in a stationary homogeneous isotropic flow. They obtained good agreement with the experimental data of Qureshi et al. (2007) and Voth et al. (2002) regarding particle acceleration variances. To the best of our knowledge, there are only few experiments with inertial particles with a notable velocity relative to the fluid in turbulent inhomogeneous shear flows. Sato \& Hishida (1996) and Suzuki et al. (2000) studied flows of glass, cellulose and ceramic particles in water with a particle to fluid mass density ratio of about 1.5 to 4 . These were channel and not pipe flows. Particle inertia and volume load effects were found to be manifest through changes of several terms in the turbulence kinetic energy equation.

Let $U_{T V}$ be the magnitude of the terminal velocity of the dispersed phase and let $u_{r m s}$ be the root-meansquare velocity of the carrier phase. A rather complex motion of bubbles in turbulent flows has been found (Sene et al., 1994; Spelt \& Biesheuvel, 1997) to result from the interaction of the turbulent flow and particle inertia when $u_{r m s} / U_{T V}=O(1)$. Entrapment of bubbles or particles in vortical flow structures and transport of dispersed phase towards the flowing edges of eddies were found to modify the mean rise velocity of bubbles in homogeneous turbulence. More recently, Aliseda \& Lasheras (2011) investigated preferential concentration of bubbles in homogenous turbulence. The reduction of the rising velocity of bubbles was shown by them to be related to higher levels of turbulence. All the above studies with a lighter 
dispersed phase (bubbles) were performed in isotropic turbulence or in plane shear flows. These studies raise expectations concerning flows with a heavier dispersed phase and terminal velocities of the same magnitude as turbulent fluctuation velocities. If not only the turbulence level but also the lift force, by definition perpendicular to the relative velocity of the particle, would be important, differences between bubbles and particles are to be expected since the relative velocity has a different sign. In addition, up-flow and down-flow would yield opposite results. In the present study solid particles slightly heavier than the carrier phase are investigated in both up-flow and down-flow in a pipe; the shape of the particles is constant, its size is well known and the bulk Reynolds number of the fluid is taken to be constant. In particular, preferential concentration, mean relative velocity and Reynolds stresses of both phases are investigated.

Aliseda \& Lasheras (2011) presented a cartoon to explain the mean relative velocity reduction of bubbles in a simplified way. They limited the interaction of bubbles and carrier fluid to $2 \mathrm{D}$ large eddies and considered the residence time of bubbles in these eddies. Adaptation of the approach of Aliseda \& Lasheras to vertical particle-laden pipe flow is straightforward and shows that the interaction of inertial particles (with $\rho_{p} / \rho_{f} \approx 1.05$ ) with relatively large turbulent flow structures leads to an increased residence time in regions where eddy velocity is upward and reduces the relative velocity, on the average. However, it is far more accurate to resolve all length and velocity scales in direct numerical simulation (DNS) and compute trajectories of inertial particles with the aid of a Lagrangian model of particle motion. This is the approach followed in the present study to investigate the mechanisms that reduce the Eulerian mean relative velocity of particles with respect to the fluid near the pipe wall. This approach increases our understanding and facilitates interpretation of experimental findings. Because of the Lagrangian numerical model used in this study, some relevant models in the literature are briefly examined below.

Attempts to model particle laden flows were done numerically in the last two decades. When particles with a size exceeding the Kolmogorov length-scale are residing in a carrier flow, the only way to fully describe the momentum exchange of the two phases is to resolve the stresses at the particle surfaces. This approach obviously requires large memory and a high processing speed. Commonly, simple geometries with a limited numbers of particles were studied; see Tryggvason et al. (2001) and Dijkhuizen et al. (2010). More recently, Picano et al. (2015) performed DNS in a turbulent channel flow with dense suspensions. Other DNS simulations utilize significant simplifications, e.g. neglect of the effect of the finite size of the particle on the flow and the assumption of a point-force approach for the particle equation of motion; see Poelma (2004). To predict trajectories in a point particle approach, the total force on a particle must be determined, which makes computation of particle trajectories in DNS possible. In the point-particle approach a lift force is usually taken into account. After the famous work of Saffman (1965) for laminar flows, Auton et al. (1988) extended the form of the lift force to high-Reynolds number flows. As Legendre \& Magnaudet (1998) have shown, the form of the lift force given by Auton et al. (1988) may be used for all particle Reynolds numbers provided that the lift force coefficient is made dependent on this Reynolds number. The analysis of the present study will focus on explanations of the trends observed experimentally and will use DNS and the point-particle approach. To be clear about this limitation, the DNS of the present study will be named PP-DNS. The use of one-way coupling in this numerical model will be validated with experimental results.

Preferential concentration of particles will be seen to play an important role. Turbophoresis, on the other hand, is a very slow process and requires a long development length. Turbophoresis is a much weaker phenomenon than radial particle transport by the lift force component that mainly depends on the derivative of the mean axial velocity with respect to the radial coordinate. Moreover, turbophoresis would yield identical concentrations in up-flow and down-flow, whereas different concentration profiles will be shown to result from this study.

Three-dimensional particle tracking velocimetry, 3D-PTV, is applied to measure fluid-particle interaction and preferential concentration for pipe flows with near-neutrally buoyant particles (with $\rho_{p} / \rho_{f} \approx$ 1.05) with low and homogeneous inlet concentration. The inertial nature of the particles is mainly due to the size, which ranges from 1.3 to 3.5 times the Kolmogorov length-scale. The choice for PTV is justified by the following reasons: the presence of two differently sized particles at the same experiment, the sparseness of the particle fields, the acquisition of simultaneous particle information at different locations across a fairly large measurement volume and the inhomogeneous nature of pipe flow which requires particle statistics collected on discrete radial positions. All flows have Reynolds number 10300, based on the bulk velocity and the pipe diameter.

The structure of the paper is as follows. In Section 2, the experimental setup is presented, including specifications of flow tracers and inertial particles, a description of the experimental conditions is given 
and the numerical method is described. In Section 3, results are presented and analyzed with the aid of PPDNS results. The PP-DNS enables the calculation of statistics of fluid velocities evaluated at the particle positions, which are relevant for analysis of the forces acting on a particle and which are virtually impossible to obtain experimentally. Focal points are inertial particle concentration profiles, Eulerian mean velocity differences and the Reynolds stresses of inertial particles. Finally, the main conclusions are stated in Section 4.

\section{Experimental Setup}

\subsection{Test rig}

Turbulent particle-laden pipe flows have been created in a water loop driven by a centrifugal pump; see figure 1. The in-line $3 \mathrm{~kW}$ centrifugal pump of type DPV18-30, manufactured by "Duijvelaar pompen", allows Reynolds numbers, based on the bulk velocity, $U_{b}$, and pipe diameter, $D$, in the range $10^{3}$ to $10^{5}$.

A set of valves is arranged in such a way that downward and upward vertical flows are possible. A frequency controller permits fine-tuning of the Reynolds number by adjusting the mass flow rate. The mass flow rate is measured by means of a Micro Motion Elite CMF300 mass flow (Coriolis meter) and mass density meter, whose inaccuracy is less than $0.5 \%$ of the registered flow rate. A water reservoir (tank I) is located at the bottom of the setup and contains about $2 \mathrm{~m}^{3}$ of water. This volume facilitates water temperature stabilization and Reynolds number control. Temperature during a test-run was essentially constant, varying by $0.1^{\circ} \mathrm{C}$ at most. Submerged pumps are placed in the reservoir tanks at the bottom and at the top (tank II, $0.15 \mathrm{~m}^{3}$ ) of the setup in order to promote homogeneous dispersion of the added tracers and inertial particles.

The measurement section consists of a glass pipe to ensure optical accessibility. A water-filled rectangular glass box around the pipe minimizes optical distortion. The pipe inner diameter is $100 \mathrm{~mm}$ and its wall thickness is $10 \mathrm{~mm}$. Flow straighteners, tube bundle conditioners of ISO 5167-1:1991, see Miller (1996), are employed to avoid secondary flows due to bends. As a result, nearly uniform fluid velocity profiles are obtained just after the flow straighteners. In up-flow, 3D-PTV measurements are performed at $45 \mathrm{D}$ downstream of the entrance section; in down-flows, at $20 \mathrm{D}$. 


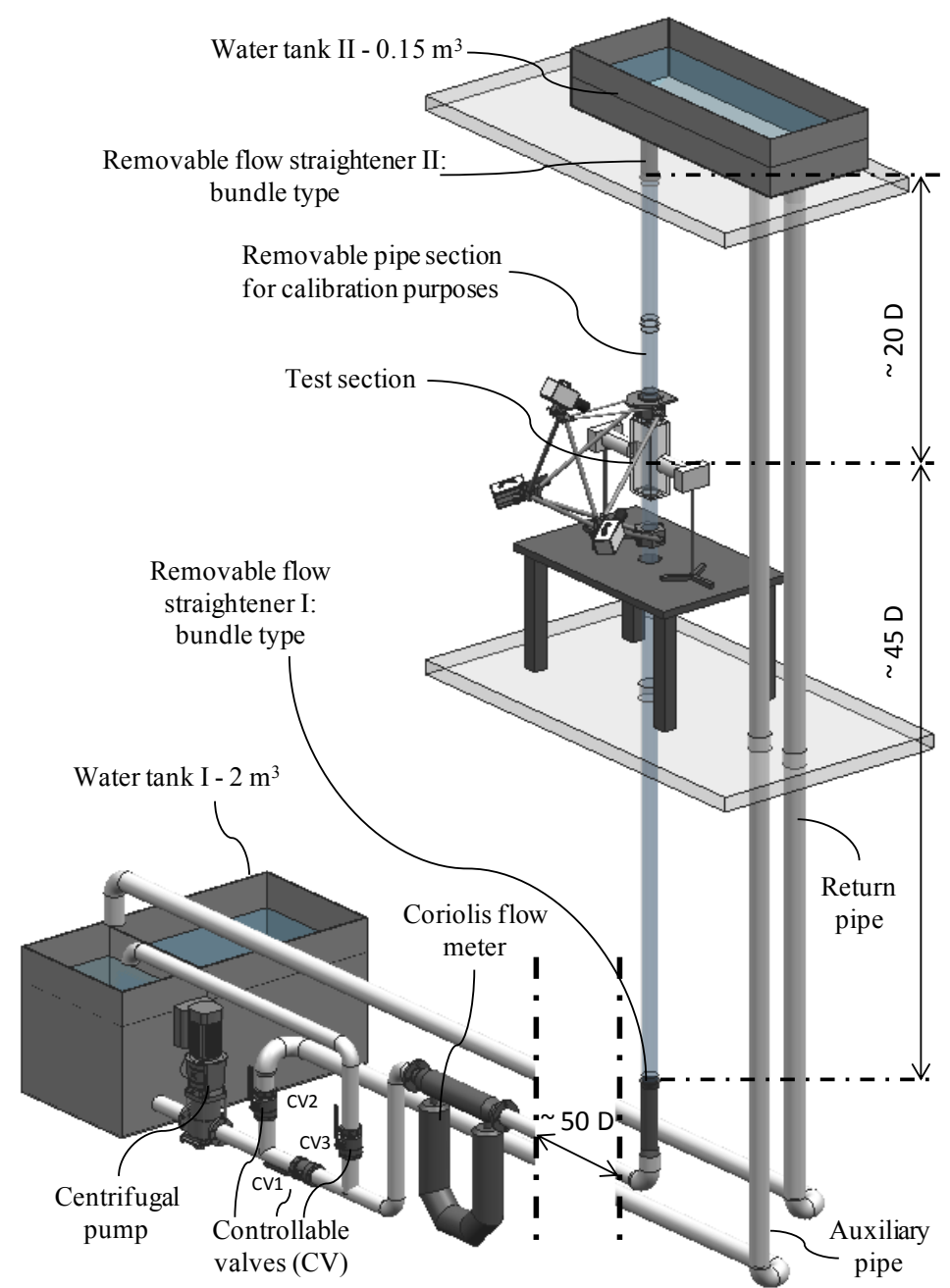

Figure 1. Schematic of the experimental 3D-PTV setup for downward or upward particle-laden pipe flow.

Three "HighSpeedStar" cameras with 12-bit grayscale CMOS sensor and a resolution of 1024 x 1024 pixels have been utilized to capture instantaneous 3D particle positions in a measurement volume of $10 \times 10 \times 10 \mathrm{~cm}^{3}$. The cameras can record at $1000 \mathrm{~Hz}$ at full resolution, but were operated at $50 \mathrm{~Hz}$ to maximize the flow measurement time. Recordings are performed until the internal memory of the cameras becomes full after approximately 2 minutes. The maximum physically relevant frequencies are about $12 \mathrm{~Hz}$ for $R e_{b}=10300$, making a $50 \mathrm{~Hz}$ sampling rate sufficient according to the Nyquist theorem. The lighting system comprised two stroboscopic light sources with an output of $5 \mathrm{~J}$ per pulse; see figure 1 . The strobes were custom-built to maximize the light output at a maximum of $60 \mathrm{~Hz}$ with a light pulse duration of approximately $40 \mu \mathrm{s}$.

\subsection{Settings and accuracy of three-dimensional particle tracking velocimetry}

While a minimum depth of field of camera and lenses must be guaranteed to obtain sharp images of moving particles in the whole volume of the measurement section, a certain field of view is needed to obtain trajectories long enough to measure all relevant flow scales. A focal length of $105 \mathrm{~mm}$, an exposure time of $20 \mu \mathrm{s}$, and a distance from the lens to the object of roughly $800 \mathrm{~mm}$ were selected. The sensor resolution is 1 pixel $^{2}=17 \mu \mathrm{m} \times 17 \mu \mathrm{m}$, which corresponds to an actual area of $100 \mu \mathrm{m} \times 100 \mu \mathrm{m}$.

To determine so-called calibration functions that correlate the pixel information of three cameras to $3 \mathrm{D}$ world coordinates, an in-situ calibration unit was designed. A calibration plate manufactured from a 2.5 $\mathrm{mm}$ thick glass plate with a single-sided coating of chromium, $150 \mathrm{~nm}$ thick, is given fixed positions at 26 locations in the pipe, interspaced at $2 \mathrm{~mm}$, with a position error of less than $1 \mu \mathrm{m}$. Only motion perpendicular to the calibration plate is possible. A 2D array of circular gaps with a diameter of $0.3 \mathrm{~mm}$ 
covers the plate; the centers are $5 \mathrm{~mm}$ apart both horizontally and vertically. Third order polynomials were found sufficient to connect the recorded pixel coordinates to actual positions; fitting errors are less than 0.05 pixel.

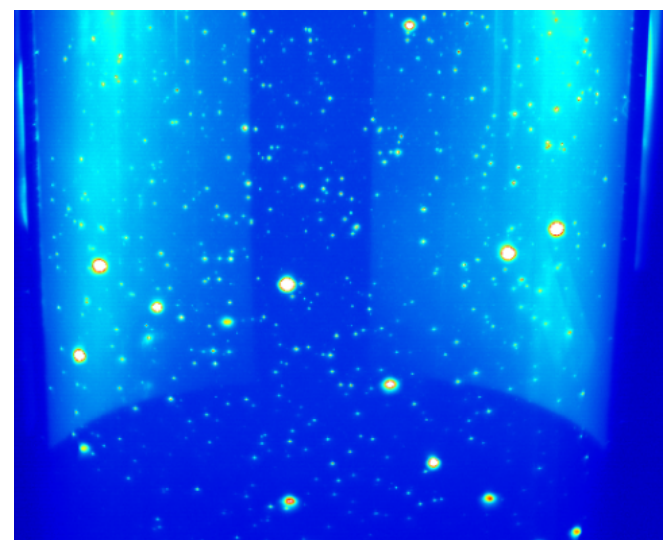

Figure 2. One out of three simultaneous recordings of particle-laden flow in the test section; frontal top camera. Inertial particles are the bigger circles.

A commercial 3D-PVT imaging code from La Vision GmbH, named Davis, has been used to obtain trajectories of tracers and inertial particles. Algorithmic details of the Davis PTV tracking code can be found in Maas (1996) and Dracos (1996). Built-in imaging filters were used to improve the contrast between tracers and background. The 2D determination of the center of a particle in the camera plane is done by a Gaussian fit. The so-called triangulation error is a measure of uncertainty of the 3D particle position. In the present measurements, the maximum triangulation error was 0.2 pixel, roughly $20 \mu \mathrm{m}$.

The particle tracking algorithm yields matrices which contain time reference and spatial positions of particle trajectories from the flow measurement images. Before the statistical analysis of turbulent pipe flow is carried out, the spatial positions are converted from Cartesian to cylindrical coordinates. In the proximity of the pipe centerline, $r / R=0$, the discontinuity of radial and tangential velocities for cylindrical coordinates can cause wrong differentiation of displacements in time. If a particle crosses $r=0$, the radial velocity, $u_{r}$, may appear to be zero, and the tangential velocity, $u_{\theta} \approx \pi \mathrm{r} / d t$; see Equations (2.1) and (2.2). This problem is avoided by employing a Cartesian frame of reference around the tube axis.

$$
\begin{aligned}
& u_{r}\left(t_{j}\right)=\left[r\left(t_{j+1}\right)-r\left(t_{j}\right)\right] / d t \\
& u_{\theta}\left(t_{j}\right)=\left[\theta\left(t_{j+1}\right)-\theta\left(t_{j}\right)\right] r\left(t_{j}\right) / d t
\end{aligned}
$$

After the coordinate transformation, the differentiation in time of the validated trajectories generates the velocity vectors. In a similar way, accelerations have been derived from smoothed velocity histories; see Oliveira et al. (2015). Velocities derived by straightforward interpolations of consecutive 3D positions of a particle trajectory have been proved reliable to obtain pipe flow statistics. The uncertainty in the magnitude of the velocity vector obtained from two consecutive positions is for both tracers and particles about $0.06 \mathrm{~mm} / \mathrm{s}$. Unrealistic trajectories are avoided by two filters: a length filter and an outliercheck of five times the standard deviation of the velocity components. The length filter consists of elimination of particle trajectories comprising less than 10 positions. Mean and maximum particle trajectories consist of 30 and 81 positions, respectively. More information about the particle tracking algorithm used to obtain trajectories of tracers and inertial particles and the trajectory analysis method of individual particle trajectories is given by Oliveira et al. (2013) and Oliveira (2012).

The number of tracer trajectories measured in the range $r / R=0.6$ to 1 decreases with increasing $r$. The difficulties in measuring tracer trajectories in this region were mainly due to light reflections stemming from the difference between the refractive indices of water, $n \approx 1.33$, and glass, $n \approx 1.51$, and the curvature of the glass pipe. Light reflections deteriorate the contrast between tracers and background. However, the fluid flow could also be measured for $r / R>0.6$ despite the lower number of usable tracers there. The measured concentration of tracers is roughly linear from $r / R=0$ to 0.6 and decreases towards the wall for $r / R>0.6$ for all flow cases. In Oliveira et al. (2013), Eulerian statistics of a single-phase turbulent pipe flow acquired by 3DPTV have been found to be trustworthy in the near-wall zone if the number of velocity vectors per radial bin exceeds 1000 . The same criterion is also applied in the present work. 
In the detection of inertial particle trajectories, the above contrast problem between particles and background did not occur. The bigger imaging projection area of inertial particles on the camera sensor, exceeding the projection of tracers by a factor of 16, avoided problems on the identification of particles; see Fig. 2. While the projection of a tracer image covers nearly an area of $2 \times 2$ pixels, the projection of an inertial particle covers $8 \times 8$ pixels. Roughly, every 3D inertial particle position identified in the tracking algorithm corresponds to a real particle. On average, approximately $3 \times 10^{5}$ inertial particle positions have been identified in each particle-laden case. 3D particle positions were identified with a camera frame rate of $50 \mathrm{~Hz}$ in average time intervals of 50 minutes. In this period of time, a water volume corresponding to $\approx 2.3 \mathrm{~m}^{3}$ crosses the test section. In this way, accurate measurements of concentration profiles of inertial particles have been obtained.

The derivative of particle trajectories with respect to time generates on average $2 \times 10^{6}$ velocity vectors for tracers and $3 \times 10^{5}$ for inertial particles in each particle-laden case. The velocity vectors are ensembleaveraged in radial bands with a width of $\Delta r=1 \mathrm{~mm}$, except for the first bin, which extend from $r=0$ to $r=$ $0.5 \mathrm{~mm}$, see Oliveira et al. (2013). A cylindrical coordinate system $(r, \theta, z)$ with origin at the pipe centerline and with the axial axis, $z$, anti-parallel to the gravitational acceleration, $g$, is used for both downand up-flow.

To check the accuracy of the mean axial velocity profiles, the products of the mean axial fluid velocity and the area of each discrete bin, $\left\langle U_{z}\right\rangle_{k} A_{k}$, were summed to obtain the mean volumetric flow rate, $Q$, through the measurement volume. Temperature measurements yielded the water mass density, $\rho$. The resulting product $\rho Q$ corresponded to the mass flow rate given by the Coriolis meter within its accuracy range.

\subsection{Properties of particles}

Properties of polystyrene particles applied in the present particle-laden experiments are given in table 1. The fluid time-scale $\tau_{\eta}$ in the Stokes number, $S t$, and the fluid length-scale $\eta$ are the Kolmogorov scales for fully developed single-phase pipe flow at $R e_{b}=10300$ as computed from DNS results by Veenman (2004). The Kolmogorov length varies between $0.6 \mathrm{~mm}$ in the pipe core and $0.2 \mathrm{~mm}$ at the wall, whereas the Kolmogorov time varies between $187 \mathrm{~ms}$ in the core and $57 \mathrm{~ms}$ at the wall. The Reynolds number based on the wall shear velocity and the pipe diameter, $R e_{\tau}$, is 647 . For evaluation of the particle timescale, $\tau_{p}$, the relaxation time for particles in stationary flow is used; see Albrecht et al. (2003):

$$
\tau_{p}=\left(d_{p}^{2} \rho_{p} / 18 \mu\right)\left(1+0.5 \rho_{f} / \rho_{p}\right)
$$

where $\mu$ is the dynamic viscosity of the fluid, $d_{p}$ is the particle diameter and $\rho_{p}$ and $\rho_{f}$ are the mass densities of particles and of fluid, respectively. A relaxation time of $\tau_{p} \approx 4 \mathrm{~ms}$ is obtained for the tracers. Note that the fluid inertia is accounted for by the added mass coefficient 0.5 which close to a wall is increased to about 0.7 ; see Van der Geld (2002).

\begin{tabular}{cccccccc}
\hline Particles & $\begin{array}{c}\text { Mass } \\
\text { density } \\
{\left[\mathrm{kg} / \mathrm{m}^{3}\right]}\end{array}$ & $\begin{array}{c}\text { Diameter } \\
d_{p}[\mathrm{~mm}]\end{array}$ & $\begin{array}{c}\text { Terminal } \\
\text { Velocity, } U_{T V}{ }^{\mathrm{a}} \\
{[\mathrm{mm} / \mathrm{s}]}\end{array}$ & ${ }^{\mathrm{a}} R e_{p}$ & ${ }^{\mathrm{b}}$ St $=\tau_{p} / \tau_{\eta}$ & ${ }^{\mathrm{b}}$ St $=\tau_{p}{ }^{+}$ & $\begin{array}{c}{ }^{\mathrm{c}} \text { Length-scale } \\
\text { ratio: }: d_{p} / \eta\end{array}$ \\
\hline Flow tracers & 1050 & 0.2 & 1.0 & 0.18 & $0.02-0.07$ & 0.10 & $0.33-1$ \\
\hline Inertial particles & 1050 & 0.8 & 10.2 & 7.76 & $0.35-1.11$ & 1.56 & $1.33-3.5$ \\
\hline
\end{tabular}

${ }^{a}$ Settling velocity of a particle in an infinite, stagnant pool of water.

${ }^{\mathrm{b}}$ Kolmogorov time-scales for a fully developed single-phase pipe flow at $R_{b}=10300$ as computed from the DNS code developed by Veenman (2004): $\tau_{\eta} \approx 187 \mathrm{~ms}$ at pipe centerline and $\tau_{\eta} \approx 57 \mathrm{~ms}$ close to the wall. The Stokes number based on the wall shear stress is given by $\tau_{p} /\left(\tau_{w} / \mu\right)=\tau_{p}{ }^{+}$, with $\tau_{w}$ the wall shear stress.

${ }^{\mathrm{c}}$ Kolmogorov length-scales for a fully developed single-phase pipe flow at $R e_{b}=10300$ as computed from the DNS code developed by Veenman (2004): $\eta \approx 0.60 \mathrm{~mm}$ at pipe centerline and $\eta \approx 0.23 \mathrm{~mm}$ close to the wall

Table 1. Properties of particles applied in the present particle-laden experiment 
The terminal velocity specified in table 1 is attained in quiescent fluid when gravitational and drag forces are in equilibrium:

$$
U_{T V}=\left\{4\left(\rho_{p}-\rho_{f}\right) d_{p} g / 3 C_{D} \rho_{f}\right\}^{0.5}
$$

where $g$ is the gravity acceleration and $C_{D}$ the drag coefficient; note that $U_{T V}$ is positive by definition. The drag coefficient is a function of the particle Reynolds number, $R e_{p}=d_{p} U_{T V} / v$, which is based on the particle diameter and the terminal velocity. In the Stokes regime, $C_{D}$ is given by (2.5). For $1<R e_{p}<1000$, Schiller \& Naumann (1935) proposed the correlation given by (2.6):

$$
\begin{aligned}
& C_{D}=24 / R e_{p}, R e_{p}<1 \\
& C_{D}=\left(24 / \operatorname{Re}_{p}\right)\left(1+1 / 6 \operatorname{Rep}^{2 / 3}\right), \quad 1<R e_{p}<1000
\end{aligned}
$$

Properties of inertial particles are such that the characteristic root-mean-square velocity representative of the turbulent carrier phase, $u_{r m s}$, and the terminal velocity of the dispersed phase, $U_{T V}$, are about the same: $u_{r m s} / U_{T V}=O(1)$.

Since the bulk flow velocity, $U_{b}$, is approximately $100 \mathrm{~mm} / \mathrm{s}$, the ratio $U_{b} / U_{T V}$ is of the order of $10^{2}$ for seeding particles, see table 1 . Since $U_{b}>>U_{T V}, \tau_{p}<\tau_{\eta}$ and $d_{p}<\eta$, the employed seeding particles work well as flow tracers. For inertial particles, the ratio $U_{b} / U_{T V}$ is of the order of $10, \tau_{p} \approx \tau_{f}$ and $d_{p}>\eta$. Therefore, inertial particles have significant inertial characteristics, as is also exhibited by the differences in Lagrangian statistical properties, auto- and cross-correlations of velocities and accelerations of tracers and inertial particles; see Oliveira et al. (2015). They showed that the decay of Lagrangian velocity correlations of the applied inertial particles takes place in shorter times than the decay of the velocity correlation of flow tracers. The fluctuating velocity of a particle is coherent with the fluid as long as it remains in a characteristic large eddy structure. However, the inertial particles applied here cannot completely follow the large fluid scales due to their inertia (mainly from their finite size) and the acceleration of the fluid volume, which is named added mass. One of the reasons for the difference in tracer and particle behavior is the so-called crossing trajectory effect, where a particle heavier than the surrounding fluid falls in an external force field, such as gravity, from one eddy to another at a rate faster than the average eddy-decay rate. In addition, particles of intermediate size in shear flows experience a hydrodynamic lift.

\subsection{Experimental conditions}

Downward and upward vertical flows have been measured at the same bulk Reynolds number based on the tube diameter, $R e_{b}$. The bulk velocity of each flow, $U_{b}$, was adapted to temperature changes to keep $R e_{b}$ $\approx 10300$. Single-phase flows serve as a reference for two-phase flows. Only tracers are employed in singlephase flow measurements. Both tracers and inertial particles are present in particle-laden measurements. A mean volume fraction of tracers less than $10^{-6}$ is applied to all experiments to prevent the enhancement of dissipation effects; see Elgobashi (1994). Particle-laden flows with mean volume fraction of inertial particles, $\left\langle\Phi_{v}\right\rangle$, ranging from $5.0 \times 10^{-6}$ to $3.2 \times 10^{-5}$ have been tested.

A particle-laden experimental case is represented here by a number indicating the flow direction (1 or 2 ) and by a letter indicating the mean concentration of inertial particles (A, B or C); see table 2. The reference single-phase flows are $1 \mathrm{~S}$ and $2 \mathrm{~S}$. Details on the single-phase pipe flow based on the scales of the current experiment and in fully developed condition (case $1 \mathrm{~S}$ ) are presented in table 3 . The variables $R e_{b}$, $R e_{\tau}, U_{b}, \eta$ and $\tau_{\eta}$ were already defined above. $U_{c}$ is the centerline velocity and $v$, the fluid kinematic viscosity. The wall shear velocity, $u_{\tau}$, was derived from DNS data provided by Veenman (2004) by implementing the actual pipe diameter. In the remainder of this work, the bulk velocity $U_{b}$ is chosen as normalization quantity instead of the wall shear velocity, $u_{\tau}$, which is often used in the literature, because $U_{b}$ can be determined more accurately in our experimental setup; see section 2.2. Single-phase flow experimental results of Oliveira et al. (2013) at $\operatorname{Re}_{b}=10,300$ were in accordance with DNS results. The significance of the pipe flow in relation to particle motion can be inferred by comparing tables 1 and 3 , and by the graphical results in section 3. The maximum rms fluid velocity occurs at $r / R \sim 0.95$ and for the streamwise direction. 


\begin{tabular}{ccc}
\hline Case & Flow Direction & $\left\langle\boldsymbol{\Phi}_{\boldsymbol{v}}\right\rangle$ \\
\hline 1S & Upward & 0 \\
1A & Upward & $0.5 \times 10^{-5}$ \\
1B & Upward & $1.4 \times 10^{-5}$ \\
1C & Upward & $3.2 \times 10^{-5}$ \\
& & \\
2S & Downward & 0 \\
2A & Downward & $1.8 \times 10^{-5}$ \\
2B & Downward & $2.8 \times 10^{-5}$ \\
\hline
\end{tabular}

Table 2. The single-phase and particle-laden flow experiments.

\begin{tabular}{|c|c|c|}
$R e_{b}$ & 10,300 & - \\
${ }^{\mathrm{a}} R e_{\tau}$ & 647 & - \\
${ }^{\mathrm{a}} u_{\tau}$ & 6.47 & {$[\mathrm{~mm} / \mathrm{s}]$} \\
$v / u_{\tau}$ & 0.15 & {$[\mathrm{~mm}]$} \\
$U_{c} / U_{b}$ & 1.28 & - \\
${ }^{\mathrm{a}} \eta$ & $0.23-0.60$ & {$[\mathrm{~mm}]$} \\
${ }^{\mathrm{a}} \tau_{\eta}$ & $187-57$ & {$[\mathrm{~s}]$} \\
${ }^{\mathrm{b}} u_{z}, \max$ & 16.6 & {$[\mathrm{~mm} / \mathrm{s}]$}
\end{tabular}

a As computed from the DNS code developed by Veenman (2004) for the pipe diameter actually used in the experiments.

${ }^{\mathbf{b}}$ Maximum rms fluid velocity. It occurs at $r / R \sim 0.95$ and for the streamwise direction.

Table 3. Details on the current single-phase pipe flow in fully developed condition for $U_{b}=100 \mathrm{~mm} / \mathrm{s}(1 \mathrm{~S})$.

\subsection{Point-particle Direct Numerical Simulation}

This paper is focused on experimental findings and the interpretation thereof. However, in order to be able to interpret some of the results, we also performed numerical simulations of particle-laden turbulent pipe flow at the same Reynolds number for up-flow. These simulations are not intended to cover the complete physics of the problem, but to complete the understanding of the experimental findings.

In a real DNS all scales of the flow around each particle should be resolved, which is computationally unfeasible with many particles. Usually, PP-DNS is performed in cases where the number of particles or the range of scales is too large, although PP-DNS is often assumed to be only valid in case the particles are small compared to the Kolmogorov scale (Bagchi \& Balachandar, 2003; Balachandar \& Eaton, 2010; Vreman, 2016), which is certainly not true in our experiments. However, since the particle volume fraction in our experiments is so small, that the effects of the particles on the continuous phase are negligible, oneway coupled PP-DNS and a force balance on each particle that includes all relevant forces exerted by the fluid on the particles will be used here. One of the advantages of the one-way coupling is that the liquid velocity and liquid vorticity at the center of the particle, needed for some of the forces on the particle, are well-defined.

The present section briefly describes the numerical method of the PP-DNS. An Eulerian-Lagrangian approach is applied, where the continuous phase is described in an Eulerian way by the incompressible Navier-Stokes equations in cylindrical coordinates and each particle is tracked in a Lagrangian way by solving its equation of motion. The numerical method for the continuous phase is the same as in Walpot et al. (2007). A pipe with a length equal to 5D is used together with periodic conditions in the axial direction. Naturally, the boundary conditions in the tangential direction are periodic as well. A Fourier-Galerkin method is applied in the two periodic directions and in the radial direction a Chebyshev-collocation method is applied, but the radial direction is divided into several elements with a Chebyshev distribution of grid 
points in each of them to avoid excessive clustering of grid points near the axis of the pipe. The nonlinear terms in the Navier-Stokes equation are calculated in Fourier space making use of the 3/2-rule to avoid aliasing. For the present bulk Reynolds number of 10,300, the numbers of grid points are 150 in the radial direction, 256 in the tangential direction and 384 in the axial direction. It has been shown by Oliveira et al. (2013) that this resolution is sufficient to obtain good agreement with experimental results for single-phase flow. Time integration is performed with a second-order accurate time-splitting method, in which the nonlinear terms are treated explicitly and the viscous and pressure terms implicitly.

At the low particle-fluid mass density ratio considered here all forces exerted by the fluid on a particle need to be taken into account, but for computational reasons we omit the Basset history force. Therefore, the equation of motion on each particle comprises the buoyancy force, pressure gradient force, added mass force, drag force and shear lift force:

$$
\begin{array}{r}
\rho_{p} V_{p} \frac{d \boldsymbol{v}_{p}}{d t}=V_{p}\left(\rho_{p}-\rho_{f}\right) \boldsymbol{g}+\rho_{f} V_{p} \frac{D \boldsymbol{U}}{D t}+\rho_{f} V_{p} C A M\left[\frac{D \boldsymbol{U}}{D t}-\frac{d \boldsymbol{v}_{p}}{d t}\right]- \\
(1 / 8) \rho_{f} C D \pi d p^{2}\left|\boldsymbol{v}_{p}-\boldsymbol{U}\right|\left(\boldsymbol{v}_{p}-\boldsymbol{U}\right)-\rho_{f} V_{p} C_{L}\left(\boldsymbol{v}_{p}-\boldsymbol{U}\right) \times \boldsymbol{\omega}
\end{array}
$$

The vorticity of the flow field, $\nabla \times \boldsymbol{U}$, is denoted by $\boldsymbol{\omega}$ and $D \boldsymbol{U} / D t$ represents the Lagrangian fluid acceleration, which is the sum of the pressure gradient acceleration and a viscous deceleration:

$$
\frac{D \boldsymbol{U}}{D t}=\frac{\partial \boldsymbol{U}}{\partial t}+\boldsymbol{U} \cdot \nabla \boldsymbol{U}
$$

The undisturbed fluid velocity, $\boldsymbol{U}\left(\boldsymbol{x}_{\boldsymbol{p}}\right)$, is by definition at the location of the center of mass of the particle, $\boldsymbol{x}_{\boldsymbol{p}}$, The coefficients $C_{A M}, C_{D}$ and $C_{L}$ denote added mass, drag and lift coefficients, respectively. Coefficient $C_{A M}$ is taken to be $0.5, C_{D}$ is computed with the Schiller \& Naumann (1935) correlation given by (2.5) and (2.6) and $C_{L}$ is given by the following correlation, valid for low Reynolds numbers $\operatorname{Re}_{s}=d_{p}{ }^{2}|\omega| \rho_{\mathrm{f}} / \mu$ (van der Geld, 1997):

$$
C_{L}=3.084 / \sqrt{ } \operatorname{Re}_{s}
$$

In order to obtain the fluid velocity, vorticity and acceleration at the particle position, tri-linear interpolation is applied. Stability problems near the pipe axis are prevented by using the equations for the Cartesian components of particle position and velocity. Also for the particles periodic conditions are applied in the axial direction. Particles collide elastically with the wall of the pipe. The number of particles tracked in the simulation is larger than in the experiments. Since only one-way coupling is applied, this does not change the results but does increase the statistical accuracy of the results.

The simulation is started from a fully-developed turbulent flow to which particles are added at random positions, uniformly distributed over the whole volume of the pipe. The initial velocity of a particle is equal to the velocity of the fluid at the position of the particle. Statistical results of the particle properties are gathered after a statistically-steady particle volume fraction distribution in the radial direction has been reached. Simulations are only performed for up-flow. For down-flow the lift force leads to a strong motion of particles toward the wall of the pipe, resulting in a locally that high particle concentration, that two-way coupling and particle collisions cannot be disregarded.

\section{Results and Discussion}

In this section the experimental results will be presented and an interpretation will be given that is also based on a comparison with PP-DNS results and experimental results for single-phase flow. Focal points are the particle volume fraction profile, the mean axial relative velocity profile and the Reynolds stress tensors of fluid and particles. First, it will be investigated to what extent the velocity statistics obtained in the measurement section corresponds to fully-developed conditions.

\subsection{Assessment of extent of flow development}

Especially for the experiments in down-flow the distance between the flow straightener and the measurement section might be too small to obtain fully developed flow in the measurement section. Therefore, we will first investigate to what extent the velocity statistics obtained for single-phase flow 
correspond to fully-developed results obtained by means of DNS (Walpot et al., 2007; Veenman, 2004) at the same Reynolds number. The diagonal components of the Reynolds stress tensor of single-phase results for up-flow will be seen to correspond well with the DNS results, which indicates that this flow is indeed fully developed; see also Oliveira et al. (2013). However, deviations up to $15 \%$ will be seen to occur in the results for down-flow, which can only be explained by a too small distance between the flow straightener and the measurement section, which equals $20 D$. The deviations are restricted to the regions close to their maxima, i.e. $0.6<r / R<0.9$. Moreover, the mean axial velocity profiles differ by only $2 \%$ at most. Although a well-known correlation predicts that the entrance length equals approximately $20 D$ in the present experiment (Hinze, 1975), this distance is apparently insufficient for a fully-developed flow in all respects. Nevertheless, the magnitude and extent of the deviations of the experimental single-phase downflow results from fully-developed flow (DNS or up-flow) are sufficiently small to discriminate important trends in the behavior of inertial particles, as will be shown in this paper. In addition, the results of the present research can be applied to validate DNS results of particle-laden flow.

Note that the development length of two-phase flow is generally larger than that of single-phase flow. Therefore, the observation that the single-phase up-flow is fully developed does not imply that the particle concentration in the corresponding two-phase flow is fully developed in the measurement section. This will be investigated in the next section.

\subsection{Volume fraction profiles of inertial particles}

In this section we will first investigate the particle volume fraction profiles for both up-flow and downflow and then give an explanation for the observed behaviour by considering the particle equation of motion. Figure 3 shows the particle volume fraction as a function of the radial coordinate for up-flow and down-flow and for all mean particle volume fractions studied. The error-bars have a size of \pm 2 times the standard error. A sample corresponds to the instantaneous volume fraction found in one measurement for a given radial position and the mean volume fraction corresponds to the time-averaged volume fraction of particles at this same location.

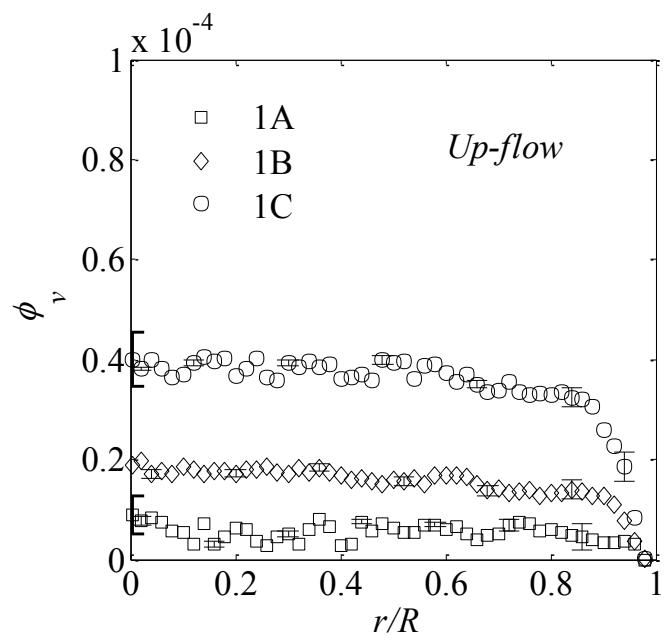

(a)

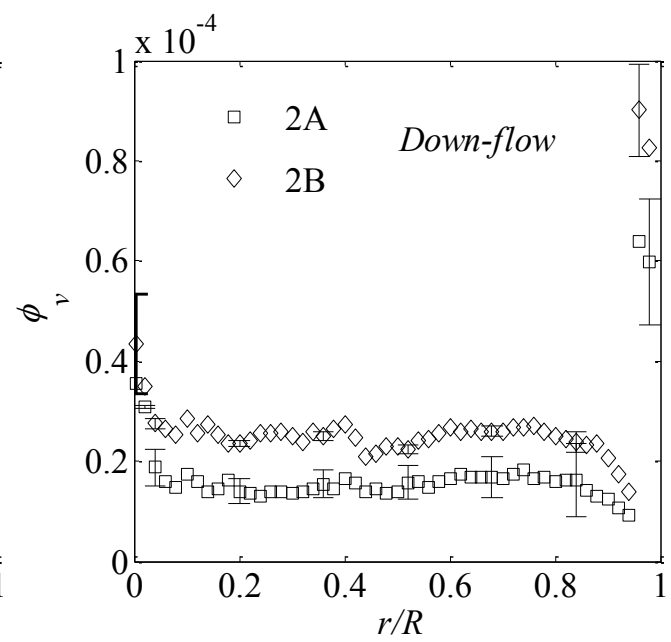

(b)

Figure 3. Volume fraction profiles of inertial particles, $\phi_{v}(r / R)$, for various values of the mean particle volume fraction. Figure 3a: up-flow, figure $3 b$ : down-flow.

The results presented in Fig. 3 are consistent: the volume fraction profiles are hardly affected even when half of the total number of samples is used. Figure 3 a shows that in up-flow the particle volume fraction is smaller close to the pipe wall, i.e. for $r / R>0.85$, than in the center. For down-flow, on the other hand, a distinct, large peak at $r / R \approx 0.97$ is observed. This shows that the volume fraction profile strongly depends on the direction of the flow, in particular in the near-wall zone. The results for down-flow also show a second peak at $r / R=0$ but with a significantly smaller magnitude than the peak near the wall. This central peak finds its origin in the development of the flow. Just after the flow straightener on top, the 
velocity profile is close to being uniform. Subsequent development of the flow requires motion towards the center. This flow carries particles along. Further downstream this peak will disappear. Note that turbophoresis, particle motion towards the wall of a pipe by the effect of turbulence, cannot explain the concentration results. If this effect was dominant, concentration profiles in up- and down-flows should be similar.

The observed differences in particle volume fraction profiles between up-flow and down-flow can be explained by the forces exerted on the particles. If the three main forces of the particle equation of motion are retained, Eq. (2.7) reduces to:

$$
0=V_{p}\left(\rho_{p}-\rho_{\mathrm{f}}\right) \mathrm{g}+(1 / 8) \rho_{f} C D \pi d p^{2}\left|\boldsymbol{v}_{p}-\boldsymbol{U}\right|\left(\boldsymbol{v}_{p}-\boldsymbol{U}\right)-\rho_{f} V_{p} C L\left(\boldsymbol{v}_{p}-\boldsymbol{U}\right) \times \boldsymbol{\omega}
$$

The main contribution from the vorticity to this equation is through the contribution to its tangential component from the mean axial velocity $d<U_{z}>/ d r$ close to the wall. The axial component of (3.1) therefore results in $v_{p, z}=U_{z}-U_{T V}$. The radial component provides a balance between lift and drag and can be simplified to $v_{p, r}=4 d_{p}\left(C_{L} / C_{D}\right) d<U_{z}>/ d r / 3$. For up-flow this radial particle velocity is directed towards the center of the pipe, whereas for down-flow it is directed towards the wall. Point particle DNS simulations without lift force resulted in uniform particle concentrations, which confirms the above explanation. Next we will use force balance (3.1) to estimate the magnitude of the mean radial particle velocity.

If only the dominant forces are retained, the radial component of (3.1) can be written as:

$$
-(1 / 8) \rho_{f} C_{D} \pi d_{p}^{2}\left|\boldsymbol{v}_{p}-<\boldsymbol{U}>_{L}\right|<v_{r e l, r}>_{\mathrm{L}}-\rho_{f} V_{p} C_{L}<v_{r e l, z}>_{\mathrm{L}} \omega_{o}=0
$$

Let $\operatorname{sgn}\left(<U_{z}>\right)$ denote the sign of the mean axial velocity component. As will be shown below, the mean value of $\left|\boldsymbol{v}_{\boldsymbol{p}}-<\boldsymbol{U}\right\rangle_{L} \mid$ is close to $U_{T V}$. We use the notation $\left\langle>_{\mathrm{L}}\right.$ to indicate a Lagrangian average, i.e., the average value taken over all particles located at a certain radial coordinate. Now suppose that an inertial particle is moving towards the center of the tube. The drift velocity of the inertial particles in radial direction can be defined as the value of $\left\langle v_{r e l,}\right\rangle_{\mathrm{L}}$ that follows from (3.2):

$$
U_{\text {drift }}=\operatorname{sgn}\left(<U_{z}>\right) 4 d_{p}\left(C_{L} / C_{D}\right) d<U_{z}>/ d r / 3
$$

If the lift force coefficient is taken to be 0.5 and the mean value of $d\left\langle U_{z}\right\rangle / d r$ for $0<r / R<0.6$ is employed, a drift velocity of $0.1 \mathrm{~mm} / \mathrm{s}$ is found, directed towards the wall for down-flow and towards the center of the pipe for up-flow. The measured mean radial particle velocity is indeed of the order of $U_{\text {drift }}$ for $0<r / R<$ 0.6 , while for $0.8<r / R<1.0$ it is systematically negative in up-flow and positive in down-flow, with absolute maxima of about $0.6 \mathrm{~mm} / \mathrm{s}$ in both cases.

Upstream of the measurement section, just behind the flow straightener, the inertial particles are still homogeneously distributed over the cross section of the pipe and no preferential concentration is present yet. At that position the mean radial fluid velocity evaluated at the particle positions is still equal to the Eulerian mean radial fluid velocity, which is equal to zero. As a result of equation (3.3), the mean radial particle velocity is directed towards the axis of the pipe for up-flow and toward the pipe wall for downflow. The measured non-zero radial particle velocity shows that the particle concentration is not yet fully developed in the measurement section.

\subsection{Mean axial velocity profiles}

Figures 4 and 5 show the mean axial velocity profiles of fluid, $\left\langle U_{z}\right\rangle$, and inertial particles, $\left\langle v_{p, z}\right\rangle$, for upand down-flow. All velocities in this paper are normalized with the bulk fluid velocity, $U_{b}$, which varies from case to case. The bulk flow velocity was adjusted to keep the bulk Reynolds number for each experiment equal to 10300. Error-bars have sizes comparable to the dimensions of symbols and are not shown. 


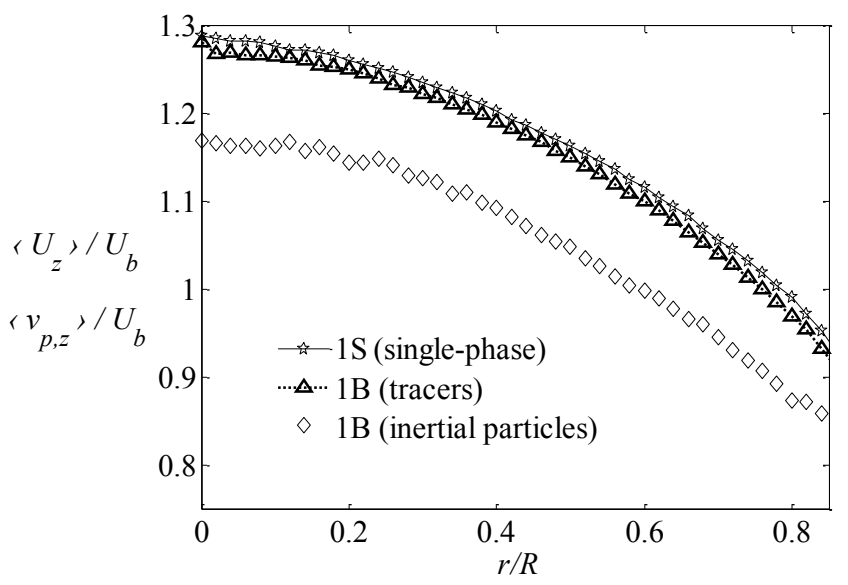

(a)

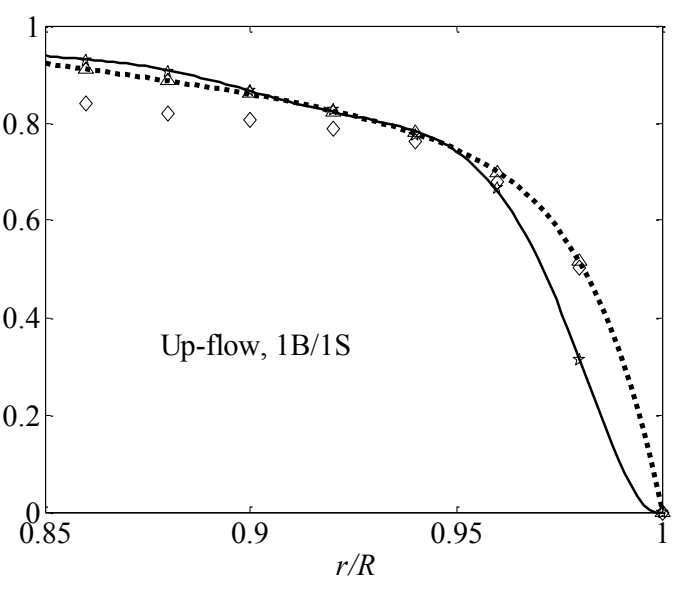

(b)

Figure 4. Mean axial velocity profiles, $\left\langle U_{z}\right\rangle$ and $\left\langle v_{p, z}\right\rangle$, of particle-laden case 1B and single-phase flow 1S (up-flow), figure $4 \mathrm{a}$ in the range $0<r / R<0.85$ and figure $4 \mathrm{~b}$ in the range $0.85<r / R<1$. The velocities are normalized by the bulk velocity of each flow, $U_{b}$. Lines are added to guide the eye. Notice the difference in scales.

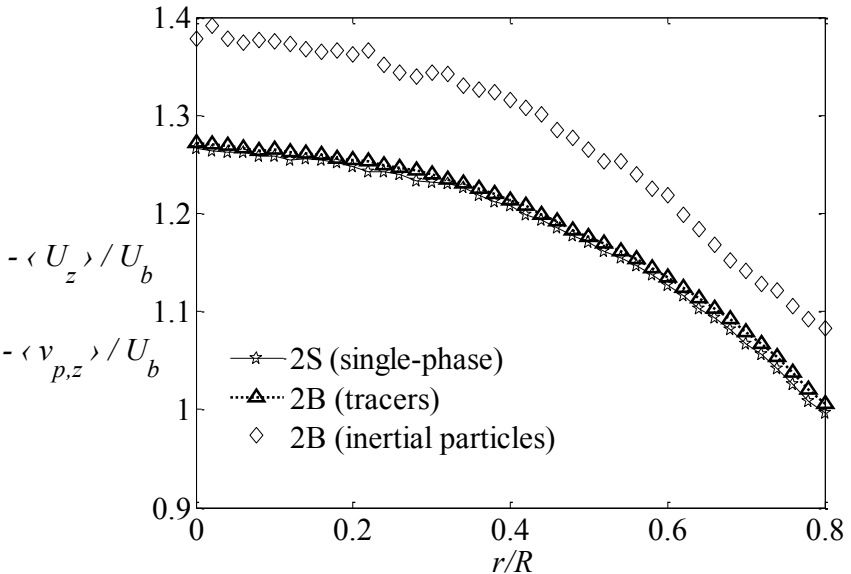

(a)

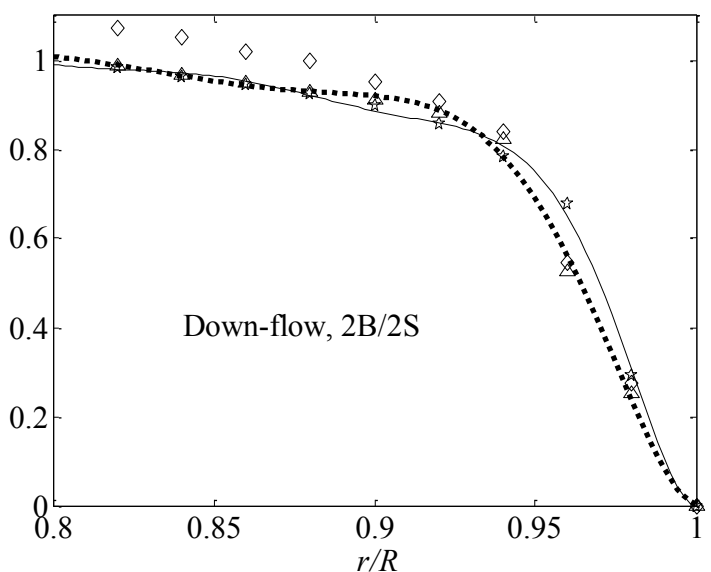

(b)

Figure 5. Mean axial velocity profiles, $\left\langle U_{z}\right\rangle$ and $\left\langle v_{p, z}\right\rangle$, of particle-laden case 2B and single-phase flow $2 \mathrm{~S}$ (down-flow), figure $5 \mathrm{a}$ in the range $0<r / R<0.8$ and figure $5 \mathrm{~b}$ in the range $0.8<r / R<1$. The velocities are normalized by the bulk velocity of each flow, $U_{b}$. Lines are added to guide the eye. Notice the difference in scales.

The mean axial fluid velocity in two-phase up-flow is slightly smaller than the corresponding singlephase velocity in most of the cross-section of the pipe. This is caused by the particles, which have a smaller velocity, dragging the fluid along. The opposite is observed in Fig. 5 for down-flow. Note that the crosssectional average fluid velocity is the same in all cases. Therefore this small difference in axial velocities is compensated close to the wall (Figs. $4 \mathrm{~b}$ and $5 \mathrm{~b}$ ).

The relative velocity is approximately constant in the range $0<r / R<0.5$. This difference is approximately equal to $10.2 \mathrm{~mm} / \mathrm{s}$, which is the value of $U_{\mathrm{TV}}$, see table 1 . The difference decreases further away from the pipe axis. For $r / R>0.90$, the mean fluid and particle velocities are almost equal, see figure 6 where the difference between the relative velocity and the terminal velocity, $U_{\mathrm{TV}}$, is shown as a function of the radial coordinate. Understanding of this finding is facilitated by a study of the PP-DNS results of the same flow. Figure 7 shows various mean axial velocity profiles obtained in the PP-DNS for up-flow. Included are the mean fluid velocity, $\left\langle U_{z}\right\rangle$, (mean is here defined as the average over time and the two homogeneous directions), the mean particle velocity, $\left\langle v_{p, z}\right\rangle$, the mean fluid velocity at the particle positions, $\left\langle U_{z}\right\rangle_{\mathrm{L}}$, and the absolute value of the mean relative velocity, which is defined as $\left\langle v_{r e l, z}\right\rangle_{\mathrm{L}}=\left\langle v_{p, z}\right\rangle-\left\langle U_{z}\right\rangle_{\mathrm{L}}$. Note that this relative velocity is not accessible in measurements, since it requires the fluid velocity at the 
particle position, which is not defined. In particle-resolved DNS this quantity is not defined as well, but for this reason we applied PP-DNS.

Figure 7 shows that if $r / R<0.7$ the difference between $\left\langle U_{z}\right\rangle$ and $\left\langle U_{z}\right\rangle_{\mathrm{L}}$ is negligible, which implies that the mean relative velocity as seen by the particle is equal to $U_{\mathrm{TV}}$. Closer to the wall the Lagrangian averaged axial fluid velocity starts to deviate from its Eulerian averaged value. Apparently, particles are preferentially located in regions where the axial fluid velocity component has a higher absolute value than the mean fluid velocity. This can be understood in the following way. Consider an inertial particle in developed upward pipe flow outside the core of the pipe. It is driven there by a positive radial fluid velocity fluctuation. Since the Reynolds stress of the fluid is positive, the axial fluid velocity fluctuation must also be positive on average. The instantaneous axial velocity of the particle, $v_{p z}$, is the sum of the constant $-U_{T V}$ and the fluid velocity, as shown by the PP-DNS results of Fig. 7. The value of $v_{p z}$ is therefore preferentially shifted to positive values of fluid velocity fluctuations. Also the lower Eulerian mean relative velocity near the wall results from this.

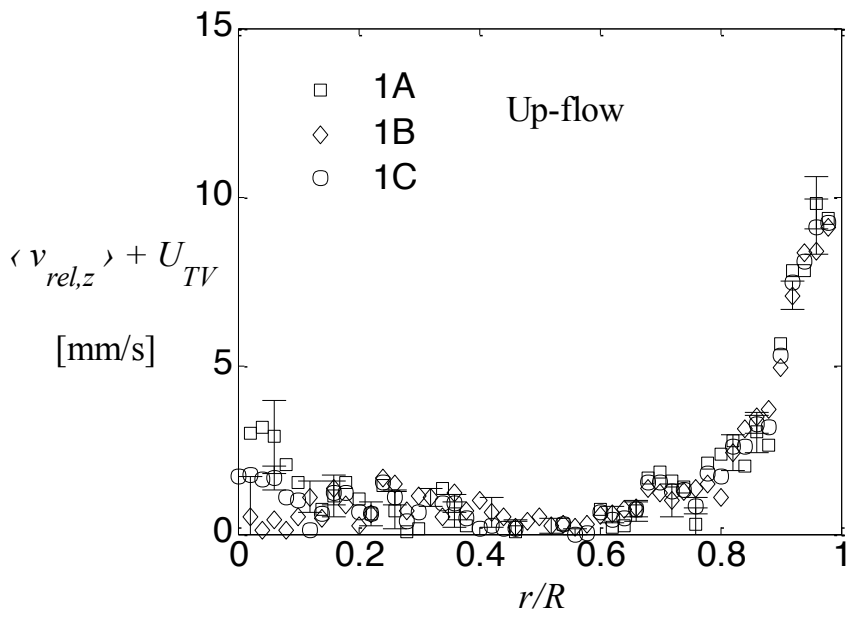

(a)

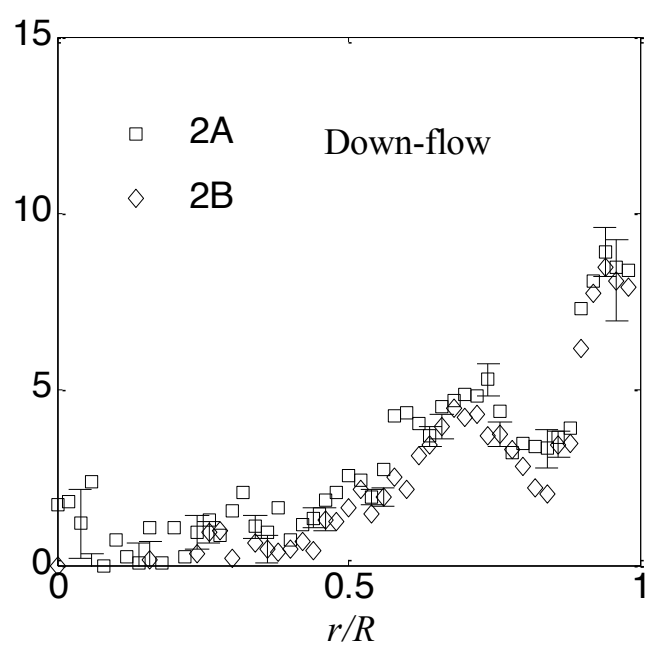

(b)

Figure 6. Difference between mean axial particle and fluid velocities, $\left\langle v_{r e l, z}\right\rangle$ for up-flow (figure $6 \mathrm{a}$ ) and down-flow (figure 6b).

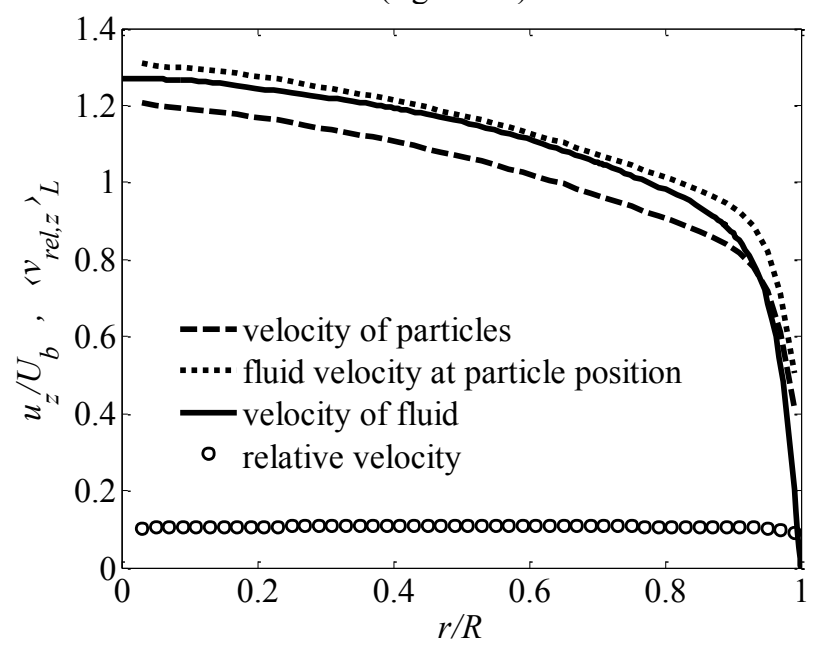

Figure 7. PP-DNS computations of local mean axial velocity components. The dashed line gives the component of the inertial particles and the dotted line the mean liquid velocity at particle position. Mean fluid velocity (solid) and the absolute value of the local mean relative velocity as sampled by the particles, $\left|\left\langle v_{\text {rel,z }}\right\rangle_{L}\right|$, (symbols) are also given.

A similar finding as in figure $6 \mathrm{~b}$ for the range $r / R<0.6$, where $-U_{T V} \approx\left\langle v_{r e l, z}\right\rangle$, was observed by Suzuki et al. (2000) in downward channel flow of water with ceramic beads with mass density exceeding the 
carrier-phase by a factor of 3.85. Ratios of RMS fluid velocity to the terminal velocity of inertial particles, $u_{r m s} / U_{T V}$, in their experiments are close to 0.2 , which is different from the value of about 1 in the present study. A maximum concentration of inertial particles occurred near the channel walls, similar as in the down-flow measurements presented here, and $\left|\left\langle v_{r e l, z}\right\rangle\right|$ was also found to decrease with decreasing distance to the wall. Suzuki et al. (2000) observed that the velocity difference between the carrier phase and the inertial particles is approximately equal to the terminal velocity. Near the wall they observed a reduction of the relative velocity of about $10 \%$ of $U_{T V}$.

In the next section it will be shown that the above explanation of the relative velocity is consistent with the interpretation of the particle Reynolds stress tensor.

\subsection{Reynolds stress tensors of fluid and dispersed phase}

In this section the components of the Reynolds stress tensor, $R_{i j}$, which are averages of the products of velocity fluctuations with $i$ and $j$ denoting cylindrical coordinates $(r, \theta, z)$, are assessed for both phases. We use $R_{i j}^{f}=\left\langle u_{i} u_{i}\right\rangle$ for the fluid phase and $R_{i j}^{p}=\left\langle v_{p}{ }^{\prime}{ }_{i} v_{p}{ }^{\prime}\right\rangle$ for the dispersed phase. In fully developed pipe flow the only decoupled direction is the tangential, which implies that all cross-correlations involving the tangential velocity are zero, which is confirmed by the experimental results.

The non-zero components of the Reynolds stress tensor are presented in figures 8,9 and 10. All results are normalized by the square of the bulk flow velocity of the corresponding experiment. In each plot, the single-phase results are also shown.

In Oliveira et al. (2013), the non-zero components of the Reynolds stress tensor of the fluid of case 1S have been shown to agree with the DNS results of Veenman (2004). While at the center of the tube $(r / R<$ $0.2)$ turbulence is nearly homogeneous, highly inhomogeneous behavior is seen closer to the wall $(0.8<$ $r / R<1$ ), see figures 8 and 9 .

Figures 8, 9 and 10 show that the results for the tracers in particle-laden flow are almost indistinguishable from single-phase flow results. This shows that the particle concentration in the experiments is so low that the particles do not significantly affect the carrier phase. Therefore, the modification of flow turbulence is not of concern in the present study due to the low concentration of particles applied. The noisy character of symbols for the Reynolds stresses of inertial particles in Figures 8, 9 and 10 can be attributed to the number of velocity samples: $2 \times 10^{6}$ velocity vectors for tracers and only $3 \times 10^{5}$ for inertial particles in each particle-laden case; see section 2.2 .

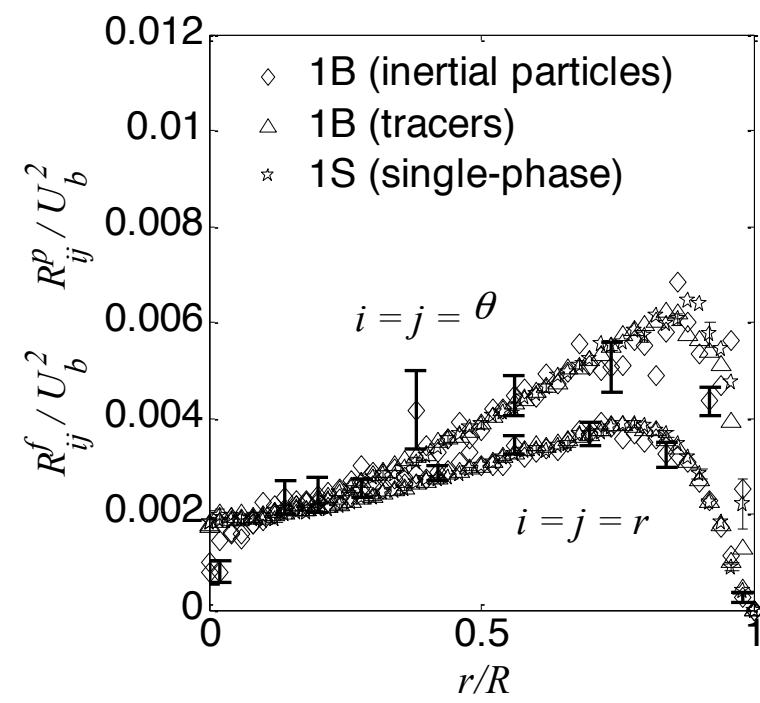

(a)

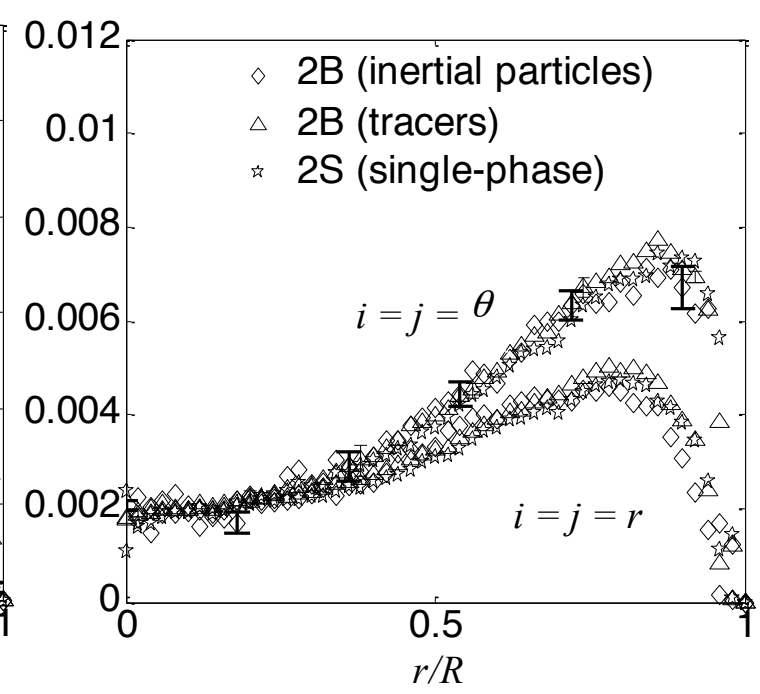

(b)

Figure 8. Radial and tangential components of the Reynolds stress tensor, $R_{r r}^{f}, R_{r r}^{p}, R_{\theta \theta}^{f}$ and $R^{p}{ }_{\theta \theta}$, for upward cases $1 \mathrm{~B} / 1 \mathrm{~S}$ (figure $8 \mathrm{a}$ ), and for downward cases $2 \mathrm{~B} / 2 \mathrm{~S}$ (figure $8 \mathrm{~b}$ ). The results are normalized by the square of the bulk velocity of each flow, $U_{b}^{2}$. Lines are added to guide the eye. 


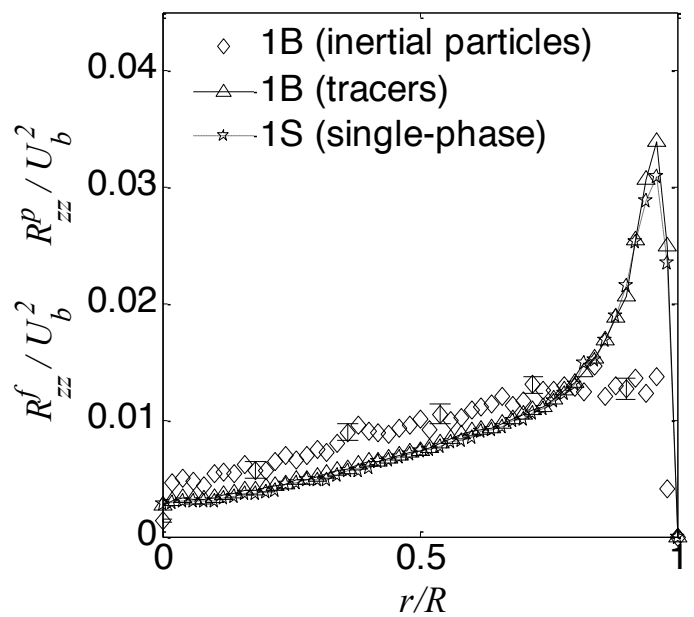

(a)

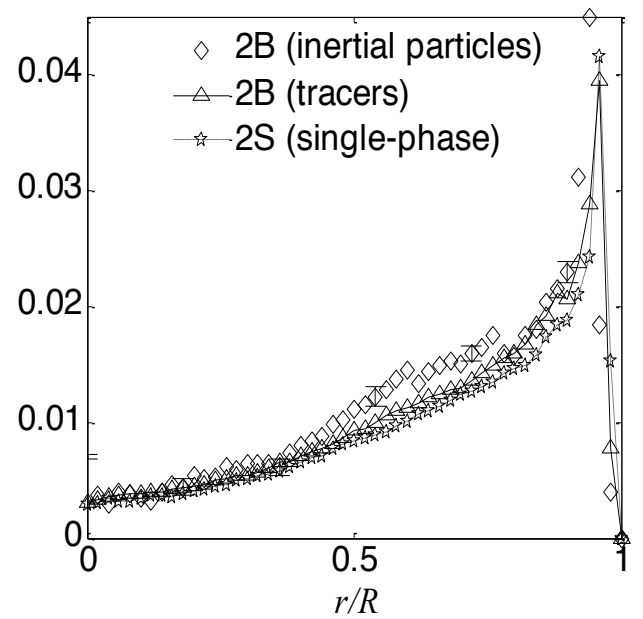

(b)

Figure 9. Axial components of the Reynolds stress tensor, $R_{z z}^{f}$ and $R_{z z}^{p}$, for upward cases 1B/1S (figure 9a), and for downward cases $2 \mathrm{~B} / 2 \mathrm{~S}$ (figure $9 \mathrm{~b}$ ). The results are normalized by the square of the bulk velocity of each flow, $U_{b}^{2}$. Lines are added to guide the eye.

The radial and tangential diagonal components of the Reynolds stress tensor of the dispersed phase are almost equal to the corresponding results for the carrier phase for both up-flow (figure 8a) and down-flow (figure $8 \mathrm{~b}$ ). However, the diagonal axial and the non-zero non-diagonal components of the Reynolds stress tensor of fluid and dispersed phase are not equal. In the range $r / R<0.8, R_{z z}^{p}$ exceeds $R_{z z}^{f}$ by more than $20 \%$ for up-flow (figure 9a) and by more than $15 \%$ for down-flow (figure 9b). For $r / R>0.8$ in up-flow both $R_{r z}$ and $R_{z z}$ for the particles are significantly smaller than for the fluid.

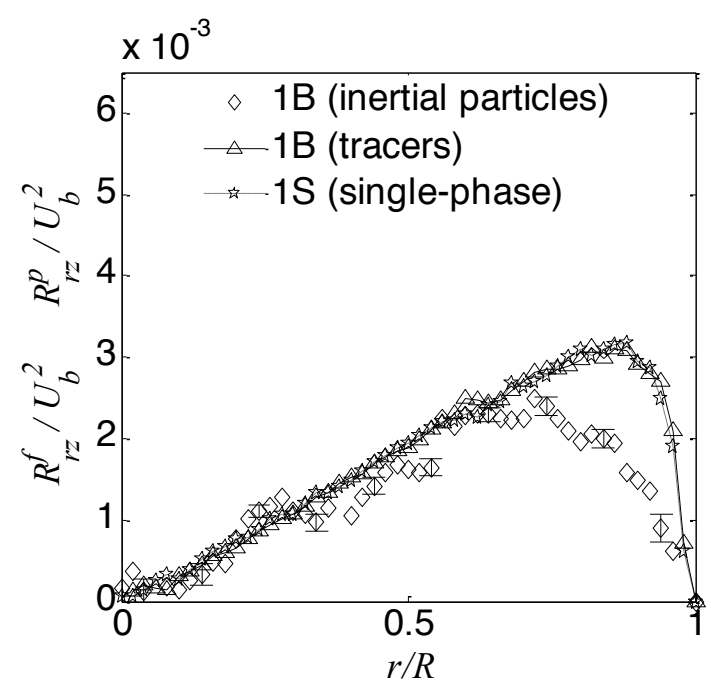

(a)

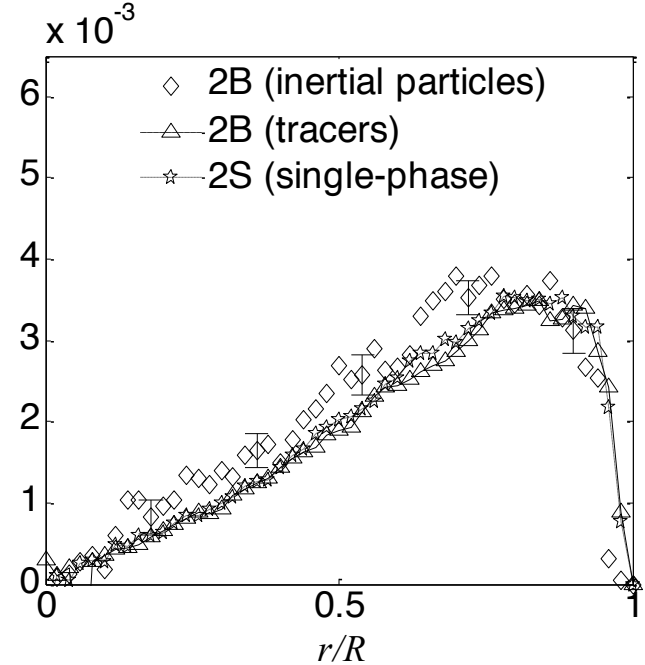

(b)

Figure 10. Non-diagonal components of the Reynolds stress tensor, $R_{r z}^{f}$ and $R_{r z}^{p}$, for upward cases 1B/1S (figure 10a), and for downward cases $2 \mathrm{~B} / 2 \mathrm{~S}$ (figure $10 \mathrm{~b}$ ). The results are normalized by the square of the bulk velocity of each flow, $U_{b}^{2}$. Lines are added to guide the eye.

In order to explain this observed behavior of the Reynolds stress tensor of the dispersed phase for $r / R>$ 0.8 , we invoke the concept of turbulent diffusion. Turbulent diffusion theory establishes a relation between the non-diagonal component of the Reynolds stress tensor and the radial derivative of the axial fluid velocity of the form:

$$
<u_{r} u_{z}>=-\Gamma_{T} \partial<U_{z}>/ \partial r
$$


The closure (3.4) is only approximate but offers a convenient way to qualitatively understand the phenomenon of turbulent diffusion. Since $d<U_{z}>/ d r<0$ in pipe flow this relation shows that $R_{r z}^{f}>0$. Next we turn to the particle Reynolds stress. Due to the direction of the lift force from the wall to the center of the pipe particles near the wall in up-flow are mainly driven by sweeps from the center to the wall. For $r / R$ $>0.7$ these particles are therefore in eddies that carry a lower absolute value of the velocity gradient $\left.d<U_{z}\right\rangle / d r$. That is why the Lagrangian sampled average of this gradient, $\left|\left\langle d U_{z} / d r\right\rangle_{L}\right|$, is smaller than the Eulerian average. The results of the PP-DNS, shown in figure 11a confirm this.

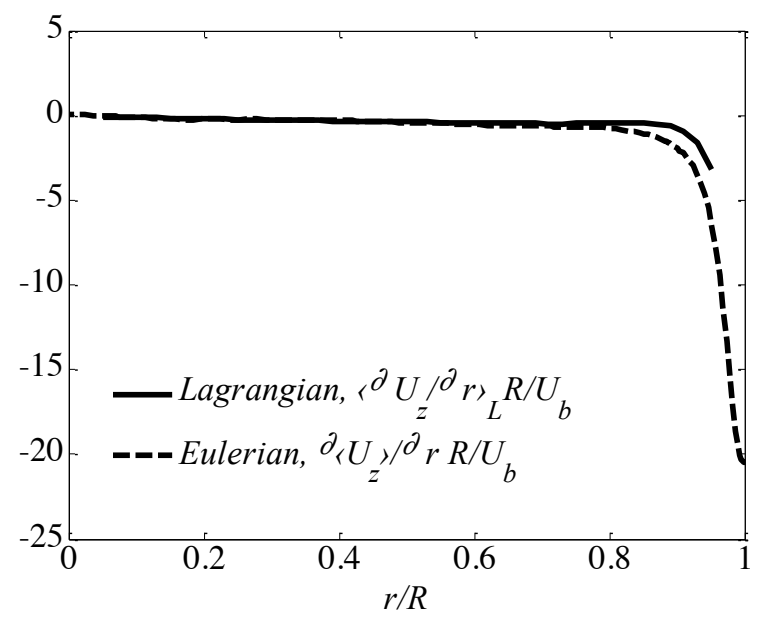

(a)

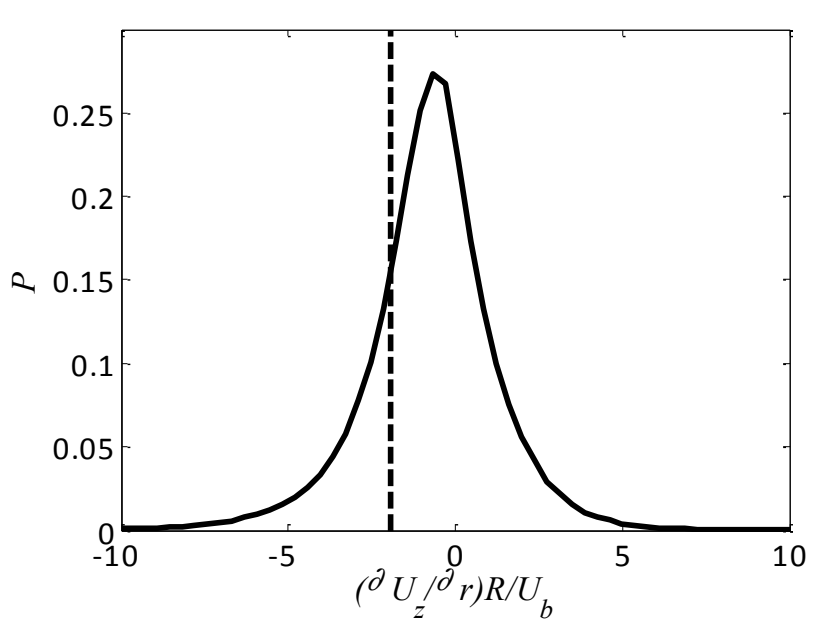

(b)

Figure 11. Comparison of $\left\langle\partial U_{z} / \partial r\right\rangle_{L}$ and $\partial\left\langle U_{z}\right\rangle / \partial r$, made dimensionless with tube radius $(R)$ and bulk velocity $\left(U_{b}\right)$, as computed by PP-DNS (a); probability density, $P$, of $\left(\partial U_{z} / \partial r\right) R / U_{b}$ (b).

The probability density function (pdf) of the Lagrangian sampled $\partial U_{z} / \partial r$ at $r / R=0.9$, shown in figure $11 \mathrm{~b}$, is a further illustration of this. This figure also shows the Eulerian average (approximately $-2 U_{b} / R$ indicated by vertical line). Diffusion theory for both the fluid and particle Reynolds stress (Eq. 3.4) now explains why $R_{r z}^{p}<R_{r z}^{f}$ close to the wall.

At the same time, the Reynolds stress $R_{z z}$ of inertial particles outside the core of the pipe is less than for fluid particles (figure 9a). This is substantiated by the PP-DNS results, which indeed showed that $\left\langle U_{z}\right\rangle_{\mathrm{L}}$ exceeds the Eulerian counterpart $\left\langle U_{z}\right\rangle$ (figure 7), while the Reynolds particle stresses are close to the experimental values, see figure 12 .

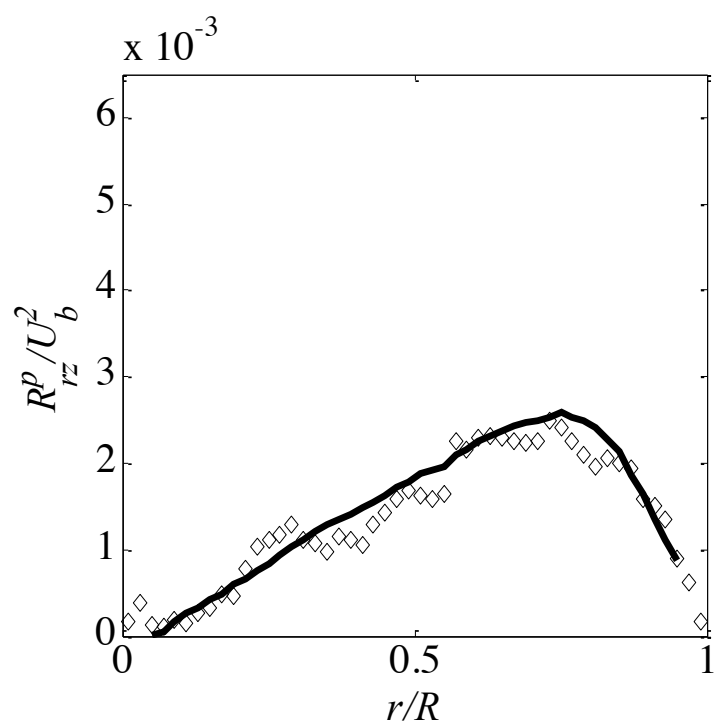

(a)

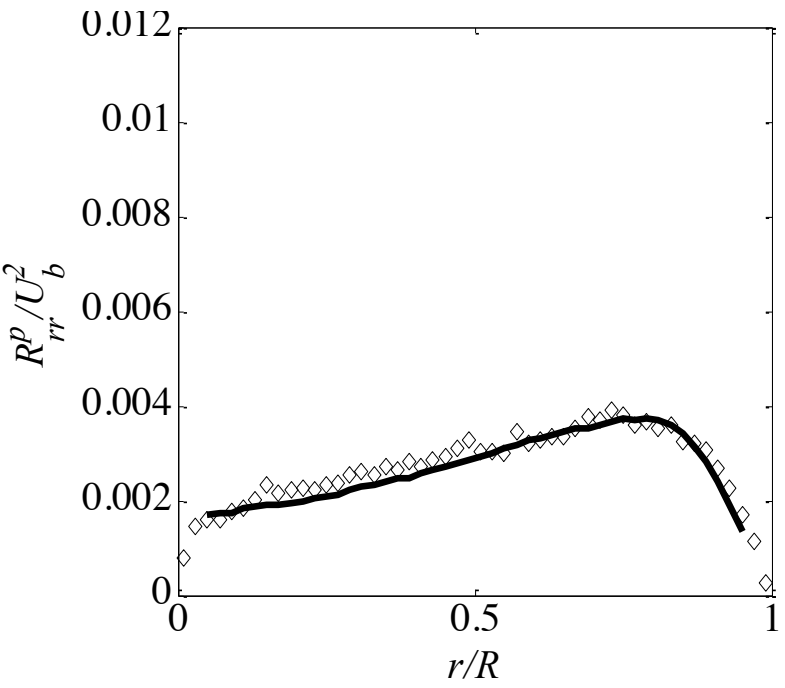

(b)

Figure 12 Reynolds stress tensor components measured in up-flow (symbols) and computed for the particles with the point force model in PP-DNS (solid line). 
In contrast to up-flow, in down-flow and close to the wall, particles are not mainly driven by sweeps from the wall to the center, since other mechanisms force particles away from the wall. Because of the large local particle concentration, diffusion is stronger near the wall than in the core. In addition, an inertial particle must also bounce at the wall. The Lagrangian average of $d U_{z} / d r$ is therefore about the same as the Eulerian average. As a consequence, the Reynolds stresses $R_{r z}^{p}$ and $R_{z z}^{p}$ of inertial particles are close to the corresponding fluid Reynolds stresses.

For $r / R<0.8$ the particle Reynolds stresses are $15 \%$ bigger (figures $9 \mathrm{~b}$ and $10 \mathrm{~b}$ ). The reason for this is probably that the down-flow case is not fully developed. This reason has been verified by the observation that upon removing the flow straightener at the inlet, as presented in Oliveira (2012), turbulent stresses become higher.

\section{Conclusions}

In this paper turbulent particle-laden pipe flow has been studied by means of 3D-PTV. We considered particles that have a slightly larger mass density than the fluid and a size larger than the Kolmogorov scale. The ratio of the RMS fluid velocity to the terminal velocity of inertial particles is of order $1 ; u_{r m s} / U_{T V}=$ $O(1)$. Results have been obtained for both up-flow and down-flow, and for both tracer and inertial particles. For up-flow the results have been compared with results of point-particle DNS, using particle-tracking and including all forces.

Two major findings were made:

- The inertial particle concentration is high near the wall in down-flow and high near the center in up-flow

- The axial component of the mean relative velocity decreases to zero near the wall in both cases.

We showed that the shear lift force is the only possible explanation for the first finding. The second finding is explained by preferential location of inertial particles in upward moving parts of near-wall eddies. Other findings concern turbulent stresses of both fluid and inertial particles and are consistent with these two explanations.

\section{Acknowledgements}

The authors gratefully acknowledge support of this work by Brazilian National Council of Research (CNPq) through the project call "Science without borders", protocol number: Proc. 405700/2013-0. Special thanks go to Prof. Júlio César Passos from UFSC, Brazil.

\section{Appendix A. State of development in up-flow and down-flow}

In this appendix we will discuss the state of development of the up-flow and down-flow measured at the test location. In Oliveira et al. (2013) it has already been shown, by comparison of velocity profile and Reynolds stress tensors with those of DNS, that the up-flow is fully developed. In the up-flow case, the distance between the flow straightener and the measurement section is $45 D$, whereas this distance is only $20 D$ for down-flow. In the down-flow case, the inlet section contains a flow straightener consisting of a set of parallel tubes; each tube has an inner diameter of $5 \mathrm{~mm}$ and a length of $40 \mathrm{~cm}$, see Fig. 13. 

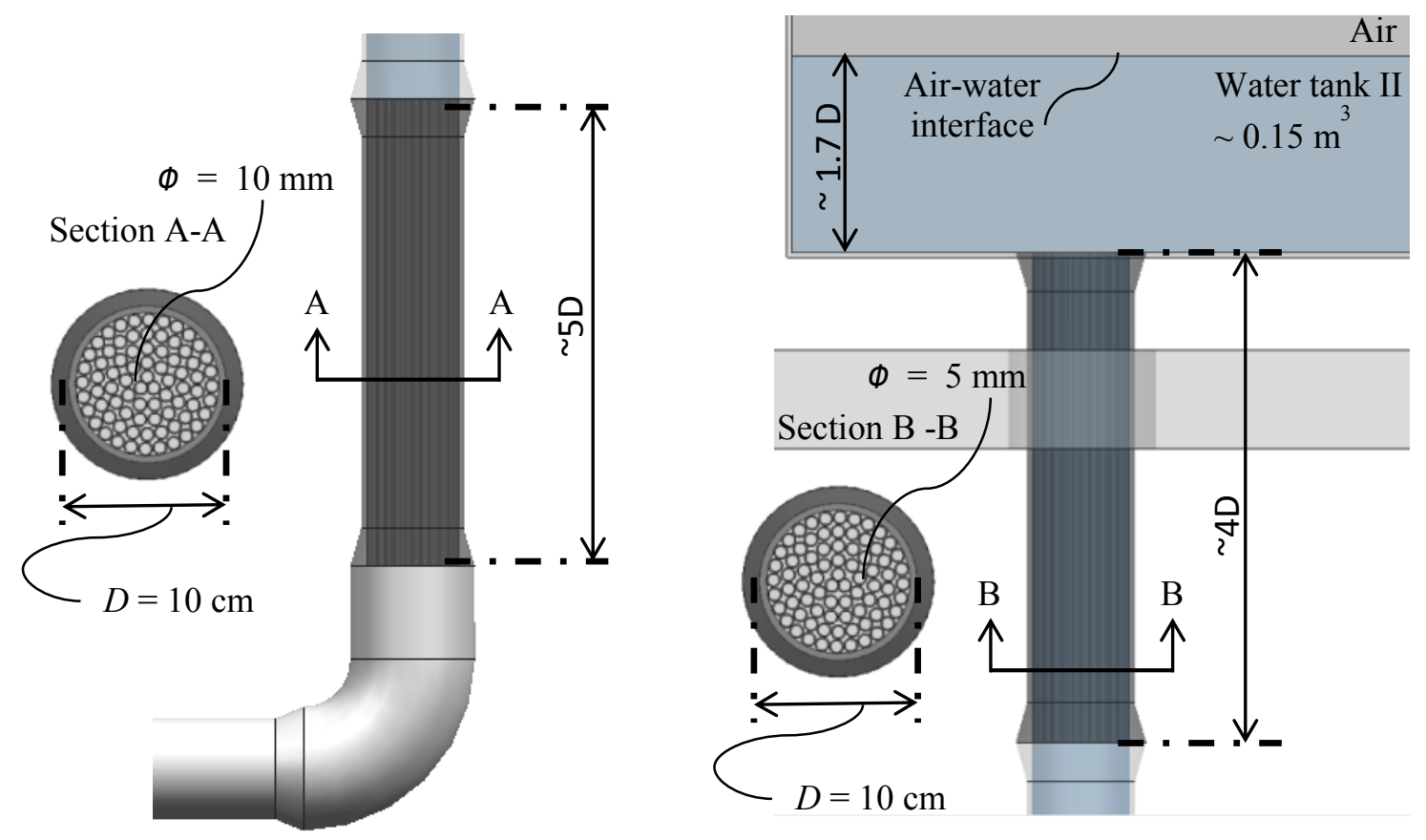

Figure 13. Schematics of flow straighteners and inlet sections for up-flow (left) and down-flow (right).

The state of development has been investigated by comparing the flow characteristics of down-flow with and without flow straightener with results from DNS and up-flow. As a typical example the $R_{z z}^{f}$ is shown for all these cases in Fig. 14. The figure shows that the up-flow results agree very well with DNS, in agreement with the conclusion in Oliveira et al. (2013). Down-flow with flow straightener results in somewhat higher velocity fluctuations, in particular in the peak close to the wall. Removal of the flow straightener leads to a significant further increase of the velocity fluctuations over the whole radius of the pipe. It is obvious that the less developed the flow is, the higher the velocity fluctuations are. The relatively small difference between the up-flow and down-flow cases with flow straightener indicates that the downflow is close to being fully developed.

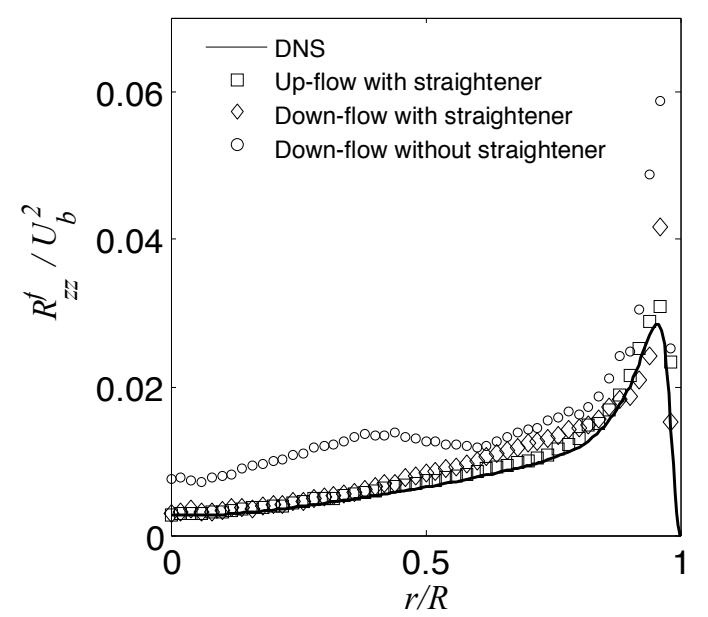

Figure 14. Comparison of axial diagonal component of the Reynolds stress tensor between DNS, up-flow and down-flow

\section{REFERENCES}

ALBRECHT, H. -E. BORYS, M. DAMASCHKE, N. \& TROPEA, C., 2003, Laser Doppler and phase Doppler measurement 
techniques. Springer-Verlag.

ALISEDA, A. \& LASHERAS, J. C. 2011 Preferential concentration and rise velocity reduction of bubbles immersed in a homogeneous and isotropic turbulent flow, Physics of Fluids, volume 23, 093301.

AUTON, T. R. 1987 The lift force on a spherical rotational flow, J. Fluid Mech., vol. 183, 199-218.

AUTON, T. R., HUNT, J. C. R. \& PRUD'HOMME, M. 1988 The force exerted on a body in inviscid unsteady non-uniform rotational flow, J. Fluid Mech., vol. 197, 241-257.

BAGCHI, P. \& BALACHANDAR, S. 2003 Effect of turbulence on the drag and lift of a particle, Physics of Fluids, volume 15, N. 11.

BALACHANDAR, S. \& EATON, J. K. 2010 Turbulent dispersed multiphase flow, Annu. Rev. Fluid Mech., Vol. 42, 111133.

BENSON, M., TANAKA, T. and EATON, J. K. 2005 The effects of wall roughness on particle velocities in a turbulent channel flow. ASME J. Fluids Eng. 127, 250-256.

BORÉE, J. \& CARAMAN, N. 2005 Dilute bidispersed tube flow: Role of interclass collisions at increased loadings, Physics of Fluids 17, 055108.

BROWN R. D., WARHAFT, Z., Voth, G. A. 2009. Acceleration Statistics of Neutrally Buoyant Spherical Particles in Intense Turbulence, Phys. Rev. Lett. 103 (19), 194501.

CAlZAVARINI, E., VOLK, R., BOURGOIN, M., LÉVÊQUE, E., PINTON, J.-F., TOSCHI, F., 2008. Acceleration statistics of finite-sized particles in turbulent flow: the role of Faxén forces, J. Fluid Mech. 630, 179-189.

CARAMAN, N., BORÉE, J. \& SIMONIN, O. 2003 Effect of collisions on the dispersed phase fluctuation in a dilute tube flow: Experimental and theoretical analysis". Physics of Fluids, vol. 15, n. 12.

DIJKHUIZEN, W. VAN SINT ANNALAND, M. \& KUIPERS, J.A.M. 2010 Numerical and experimental investigation of the lift force on single bubbles, Chemical Engineering Science, Volume 65, 1274-1287.

DRACOS, T., 1996. Particle tracking in three-dimensional space. In: Dracos, Th. (Ed.), Three-dimensional velocity and Vorticity Measuring in Image Analysis Techniques. Kluwer Academic Publishers.

ELGHOBASHI, S., 1994, On Predicting Particle-Laden Turbulent Flows, Applied Scientific Research 52: 309-329.

HINZE, J. O. 1975 Turbulence, McGraw-Hill.

KULICK, J. D., FESSLER J. R. \& EATON, J. K. 1994 Particle response and turbulence modification in fully developed channel flow. J. Fluid Mech. 277, 109-134.

KUNKEL, ALLEN, J. J. \& SMITS, A. J. M. 2007 Further support for Townsend's Reynolds number similarity hypothesis in high Reynolds number rough-wall pipe flow. Physics of Fluids 19, 055109.

KUSSIN, J. \& SOMMERFELD, M. 2002 Experimental studies on particle behaviour and turbulence modification in horizontal channel flow with different wall roughness. Exp. Fluids 33, 143-159.

LEGENDRE, D. \& MAGNAUDET, J. 1998 The lift force on a spherical bubble in a viscous linear shear flow, J. Fluid Mech. 368, 81-126.

LUCAS, D. \& TOMIYAMA, A. 2011 On the role of the lateral lift force in poly-dispersed bubbly flows, International Journal of Multiphase Flow Vol. 37, 1178-1190.

MAAS, H.-G., 1996. Contributions of digital photogrammetry to 3D-PTV. In: Dracos, Th. (Ed.), Three-dimensional Velocity and Vorticity Measuring in Image Analysis Techniques. Kluwer Academic Publishers.

MAZZITELLI, I. M., LOHSE, D. \& TOSCHI, F. 2003 On the relevance of the lift force in bubbly turbulence, J. Fluid Mech. $488,283-313$.

MILLER, R. W., 1996, Flow Measurement Engineering Handbook, McGraw-Hill, 3rd edition.

MINIER, J.-P., 2015 On Lagrangian stochastic methods for turbulent polydisperse two-phase reactive flows, Progress in Energy and Combustion Science, 50, 1-62.

OLIVEIRA, J. L. G., 2012, 3D-PTV of particle-laden turbulent pipe flows, Ph.D. thesis, Technische Universiteit Eindhoven.

OLIVEIRA, J. L. G., VAN DER GELD, C. W. M. \& KUERTEN, J. G. M., 2013, Lagrangian and Eulerian statistics of pipe flows measured with 3D-PTV at moderate and high Reynolds numbers, Flow, Turbulence and Combustion, 91 (1), 105-137.

OLIVEIRA, J. L. G., VAN DER GELD, C. W. M. \& KUERTEN, J. G. M. 2015 Lagrangian velocity and acceleration statistics of fluid and inertial particles measured in pipe flow with 3D particle tracking velocimetry.”, Int. J. Multiphase Flow 73, 97-107.

PARIS, A. D. \& EATON, J. K. 2001 Turbulence attenuation in a particle-laden channel flow. Tech. Rep. TSD-137. Stanford University.

PICANO, F., BREUGEM, W.-P. \& BRANDT, L. 2015 Turbulent channel flow of dense suspensions of neutrally buoyant spheres, J. Fluid Mech. 764, 463-487.

POELMA, C. 2004 Experiments in particle-laden turbulence - simultaneous particle/fluid measurements in grid-generated turbulence using particle image velocimetry Ph.D. thesis, Delft University of Technology.

POPE, S. B., 2000 Turbulent Flows, Cambridge University Press, Cambridge.

QURESHI, N. M., BOURGOIN, M., BAUDET, C., CARTELLIER, A. \& GAGNE, Y. 2007. Turbulent transport of material particles: An experimental study of finite size effects. Phys. Rev. Lett. 99 (18), 184502.

SAFFMAN, P. G. 1965 The lift on a small sphere in a slow shear flow. J. Fluid Mech. 22, 385-400.

SATO, Y. \& HISHIDA, K. 1996 Transport process of turbulence energy in particle-laden turbulent flow. Intl J. Heat Fluid Flow 17, 202-210.

SCHILLER, L. \& NAUMANN, Z., 1935 A drag coefficient correlation. Z. Ver. Deutsch. Ing., 77.

SENE, K. J., HUNT, J. C. R. \& THOMAS, N. H., 1994 The role of coherent structures in bubble transport by turbulent shear flows. J. Fluid Mech. 259, 219-240.

SPELT, P. D. M. \& BIESHEUVEL, A., 1997 On the motion of gas bubbles in homogeneous isotropic turbulence”, J. Fluid. Mech., 336, 221-244. 
SUZUKI, Y., IKENOYA, M. \& KASAGI, N., 2000 Simultaneous measurements of fluid and dispersed phases in a particleladen turbulent channel flow with the aid of 3-D PTV", Experiments in Fluids, 185-193.

TRYGGVASON, G., BUNNER, B., ESMAEELI, A., JURIC, D., AL-RAWAHI, N., TAUBER, W., HAN, J., NAS, S. \& JAN, Y.-J. 2001 A Front-Tracking Method for the Computations of Multiphase Flow, Journal of Computational Physics 169, 708-759.

TSUJI, Y. \& MORIKAWA, Y. 1982 LDV measurements of an air-solid two-phase flow in a horizontal pipe. J. Fluid Mech., $120,385-409$

TSUJI, Y., MORIKAWA, Y. \& SHIOMI, H. H. 1984 LDV measurements of an air-solid two-phase flow in a vertical pipe. J. Fluid Mech. 139, 417-434.

VAN DER GELD, C.W.M. 1997 Measurement and prediction of solid sphere trajectories in accelerated gas flow, Int. Journal of Multiphase Flow, 23(2), 357-376.

VAN DER GELD, C.W.M. 2002 On the motion of a spherical bubble deforming near a plane wall, Journal of Engineering Mathematics, Volume 42, N. 2, 91-118.

VEENMAN, M. P. B., 2004 Statistical analysis of turbulent pipe flow: A numerical approach. Ph.D. thesis, Technische Universiteit Eindhoven.

VOLK, R., CALZAVARINI, E., LÉVÊQUE E. \& PINTON, J.-F. 2011 Dynamics of inertial particles in a turbulent von Kármán flow, J. Fluid. Mech. 668, 223-235.

VOTH, G. A., LA PORTA, A., CRAWFORD, A. M., ALEXANDER, J. \& BODENSCHATZ, E. 2002. Measurement of particle accelerations in fully developed turbulence. J. Fluid Mech. 469, 121-160.

VREMAN, A.W. 2016 Particle-resolved direct numerical simulation of homogeneous isotropic turbulence modified by small fixed spheres, J. of Fluid Mech. 796, 40-85.

WALPOT, R.J.E., VAN DER GELD, C.W.M. \& KUERTEN, J.G.M. 2007 Determination of the coefficient of Langevin models for inhomogeneous turbulent flows by 3D PTV and DNS, Phys. Fluids 19, 045102.

YANG, T. S. \& SHY, S. S. 2005 Two-way interaction between solid particles and homogeneous air turbulence: particle settling rate and turbulence modification measurements. J. Fluid Mech. 526, 171-216. 